\title{
Using Hybrid Implicit Monte Carlo Diffusion to Simulate Gray Radiation Hydrodynamics
}

\author{
Mathew A. Cleveland*, Nick Gentile \\ Lawrence Livermore National Laboratory, L-405, P. O. Box 808, Livermore California 94550
}

\begin{abstract}
This work describes how to couple a hybrid Implicit Monte Carlo Diffusion (HIMCD) method with a Lagrangian hydrodynamics code to evaluate the coupled radiation hydrodynamics equations. This HIMCD method dynamically applies Implicit Monte Carlo Diffusion (IMD) [1] to regions of a problem that are opaque and diffusive while applying standard Implicit Monte Carlo (IMC) [2] to regions where the diffusion approximation is invalid. We show that this method significantly improves the computational efficiency as compared to a standard IMC/Hydrodynamics solver, when optically thick diffusive material is present, while maintaining accuracy. Two test cases are used to demonstrate the accuracy and performance of HIMCD as compared to IMC and IMD. The first is the Lowrie semi-analytic diffusive shock [3]. The second is a simple test case where the source radiation streams through optically thin material and heats a thick diffusive region of material causing it to rapidly expand. We found that HIMCD proves to be accurate, robust, and computationally efficient for these test problems.
\end{abstract}

\section{Introduction}

Many radiation hydrodynamic problems, such as those found in the fields of astrophysics and inertial confinement fusion, are composed of strongly heterogeneous materials that can vary in optical thicknesses by many orders of magnitude. A Hybrid Implicit Monte Carlo Diffusion (HIMCD) solver is developed in this work that can accurately resolve the radiation hydrodynamics equations more efficiently than the standard Implicit Monte Carlo (IMC) [2] approach typically used on these types of problems.

IMC is a robust method that is used to evaluate the radiative transfer equation and the associated material energy balance equation. IMC relies on a first order expansion of the change in material temperature in time to create a set of equations that are evaluated using a Monte Carlo tracking algorithm. This approach creates an additional term in the transport equation, typically referred to as "effective scattering", which physically accounts for photons being absorbed and subsequently re-emitted by the material over a time step. Effective scattering can make Monte Carlo prohibitively expensive when

${ }^{*}$ Corresponding author. Tel + 1925 422-0287; e-mail: cleveland7@llnl.gov LLNL-JRNL-639895

Preprint submitted to Elsevier

February 19, 2015 
opaque materials are present. As a result, many methods have been developed to accelerate the solution of these equations in opaque materials $[1,4,5]$.

Asymptotic analysis has shown that the diffusion approximation can accurately approximate the radiative transport solution in a thick diffuse limit [6]. Implicit Monte Carlo Diffusion (IMD) [1] can be used to accurately simulate the solution to the radiative transfer equation in regions where this limit is valid at a reduced cost compared to IMC $[1,7]$. IMD evaluates a spatial discretized diffusion equation using a Monte Carlo technique. There is a similar method in the literature known as Discrete Diffusion Monte Carlo (DDMC) [8] and differs primarily in the way that the photons are tracked in time. IMD tracks photons discretely in time based on the temporal discretization, and DDMC tracks photons continuously in time [7]. This work focuses on IMD but the reader should note that much of what is discussed is similarly applicable to DDMC. Similarly, many of the tools developed to implement this work were originally developed for DDMC and will be referenced as such.

In this work we will describe how HIMCD can be coupled to a Lagrangian hydrodynamics code to accurately resolve the fluid frame radiation hydrodynamics equations. This approach details how to use operator splitting to evaluate the material motion effects on the radiation field. We show that this operator splitting approach accurately resolves the coupled solution in diffusive radiation problems such as the Lowrie radiation hydrodynamics shock problem [3]. We also show that the coupled HIMCD/Hydrodynamics code can accuratly and efficiently, as compared to standard IMC, resolve problems that have optically thick and optically thin materials with a simple two material ablation problem.

\subsection{Hybrid Implicit Monte Carlo Diffusion}

Significant improvements in problem solution times have been demonstrated for HIMCD and Hybrid IMC/DDMC in problems that contain both thick diffusion and transparent streaming media [1,5]. HIMCD accelerates the time to solution of an IMC radiative transfer calculation. This acceleration is achieved by applying the well known diffusion approximation, using IMD, in regions of a problem that are dominated by isotropic scattering making diffusion an accurate approximation to transport. In diffusive regions, IMC particles take many short paths that can dominate the solution time. The IMD method approximates many short IMC paths with a single, much larger, path [1].

IMD is an ideal choice to combine with IMC, because of some important shared characteristics. The biggest advantage is that IMD takes advantage of similar data types used in IMC, Monte Carlo particles, that can be advanced in a similar Monte Carlo transport routine. Interface conditions can also be seamlessly integrated during a simulation allowing Monte Carlo particles to move between IMC and IMD regions. Densmore et al. [9] have developed a rigorous interface condition that will preserve the analytic emissivity of a face that separates DDMC and IMC regions. We will apply this interface condition for the IMD-IMC interface in HIMCD. IMC and IMD both apply the Fleck and Cummings [2] first order expansion of the emission source term, which creates a semi-implicit set of equations, avoiding the need for a non-linear iteration. IMD has been demonstrated to treat continuous frequency distributions similarly to IMC [7]. More recently DDMC has been demonstrated to perform well in frequency dependent problems using frequency integrated groups [10]. 
To the authors knowledge neither IMD or DDMC have previously been applied to the radiation hydrodynamics problems, and the remainder of this work will outline how HIMCD can be applied to such problems.

\subsection{Radiation Hydrodynamics}

This work implements the "fluid-frame" semi-implicit Lagrangian-frequency-independent radiation hydrodynamics equations. This means that the reference frame is considered to share the local fluid velocity and the radiation quantities are defined in reference to it [11]. This work can be extended to frequency dependent problems as in the work of Densmore et al. [10]. The equations presented here are for a single material, which is all that is required for Lagrangian Hydrodynamics.

It is an open question as to whether the fluid-frame IMC and IMD equations are strictly conservative. To determine this it is necessary to transform the equations into the lab frame and prove that these transformed equations are conservative. Preliminary work presented by Morel [12] suggests that the fluid frame diffusion equation is only conservative in the equilibrium diffusion limit.

\subsubsection{The implicit Monte Carlo radiation hydrodynamics equation}

There are four different coupled non-linear equations that comprise the set of frequency independent radiation hydrodynamics equations [11]. Variable dependencies will be defined in the text; however, they will be left out of the main equations for brevity. The first of the four radiation hydrodynamics equations expresses the time evolution of mass:

$$
\frac{D \rho}{D t}+\rho \bar{\nabla} \cdot \bar{u}=0
$$

where $\bar{u}(\bar{r}, t)[\mathrm{cm} / \mathrm{s}]$ is the material velocity at position $\bar{r}[\mathrm{~cm}]$ and time $t[\mathrm{~s}]$, and $\rho(\bar{r}, t)$ $[\mathrm{g} / \mathrm{cc}]$ is the density of the material. Here we have written the equations in terms of Lagrangian derivative:

$$
\frac{D}{D t}=\frac{\partial}{\partial t}+\bar{u} \cdot \nabla
$$

The second equation expresses the time evolution of the material kinetic energy:

$$
\rho \frac{D \bar{u}}{D t}+\bar{\nabla} p-\kappa \bar{F}=0
$$

where $\kappa \bar{F}$ [erg/ $/ \mathrm{cm}^{3}$-s] is the momentum deposited in the material by the radiation field, $\kappa(\rho, T)[1 / \mathrm{cm}]$ is the material opacity, $\bar{F}(\bar{r}, t)=\int_{0}^{4 \pi} \bar{\Omega} I(\bar{r}, t, \bar{\Omega}) d \bar{\Omega}$ [ergs $/ \mathrm{cm}^{2}$-s] is the radiation flux, $p(\rho, T)\left[\mathrm{N} / \mathrm{cm}^{2}\right]$ is material pressure, and $I(\bar{r}, t, \bar{\Omega})$ [ergs $/ \mathrm{cm}^{2}$-s-steradian] is the radiation intensity moving in the direction $\bar{\Omega}$.

The third equation expresses the time evolution of the internal material energy:

$$
\rho \frac{D \epsilon}{D t}+p \bar{\nabla} \cdot \bar{u}=c \kappa f E-\kappa f a c T^{4} .
$$

where we have applied the Fleck and Cummings time discretization giving rise to the Fleck factor [2]:

$$
f=\frac{1}{1+\frac{c \Delta t \sigma 4 a T^{3}}{\rho C v}}
$$


where $C v$ is the the materials specific heat. The remaining variables and constants in Eq. 4 are: the radiation energy density $E=\frac{1}{c} \int_{0}^{4 \pi} I(\bar{r}, t, \bar{\Omega}) d \bar{\Omega}\left[\mathrm{erg} / \mathrm{cm}^{3}\right]$ and temperature $T(\bar{r}, t)[\mathrm{keV}]$, the radiation constant $a$, and the speed of light $c[\mathrm{~cm} / \mathrm{s}]$. The internal material energy equation is operator split such that the radiation terms can be evaluated independently from the hydrodynamic terms. This results in two separate equations, one evaluating the change in material energy due to hydrodynamics:

$$
\rho \frac{D \epsilon}{D t}+p \bar{\nabla} \cdot \bar{u}=0
$$

and one evaluating the change due to absorption and emission of radiation:

$$
\rho \frac{\partial \epsilon}{\partial t}=c \kappa f E-\kappa f a c T^{4} .
$$

The material equation of state provides the relations $\epsilon(\rho, T)$ and $p(\rho, T)$ and closes the system of equations. The hydrodynamics solver evaluates Eq. 6 and the radiation solver evaluates Eq. 7. The radiation field can be described using the Fleck and Cummings semi-implicit radiation equation [2]:

$$
\begin{aligned}
\frac{1}{c} \frac{D I}{D t} & +\bar{\Omega} \cdot \bar{\nabla} I-\frac{1}{c} \bar{\Omega} \cdot \bar{\nabla} \bar{u} \cdot(\overline{\bar{l}}-\bar{\Omega} \bar{\Omega}) \cdot \bar{\nabla}_{\bar{\Omega}} I+\frac{3}{c} \bar{\Omega} \cdot \bar{\nabla} \bar{u} \cdot \bar{\Omega} I \\
& =\frac{1}{4 \pi} \kappa f a c T^{4}-\kappa I+\frac{1}{4 \pi} \int_{0}^{4 \pi} \kappa(1-f) I d \bar{\Omega}^{\prime}
\end{aligned}
$$

for the fluid frame radiation hydrodynamics equations [11]. In this equation $\overline{\bar{l}}$ is a unit matrix, $\bar{\Omega}$ is the fluid frame photon direction, $\bar{\nabla}_{\bar{\Omega}}$ is the angle gradient, and $E(\bar{r}, t)=$ $\frac{1}{c} \int_{0}^{4 \pi} I(\bar{r}, t, \bar{\Omega}) d \bar{\Omega}[\mathrm{erg} / \mathrm{cc}]$ is the frequency independent radiation energy density. The third and fourth terms, which involve gradients in the velocity, arise from the fact the the equation is written in the fluid frame. Since $\bar{\Omega}$ and the photon energy are constant in the lab frame, these quantities vary in the fluid frame as the fluid velocity varies. The third term in Eq. 8 is the aberration correction [11], which accounts for the change in the fluid frame angle. The fourth term in Eq. 8 describes the change in the fluid frame photon energy. (See [11] and [13] for a more detailed discussion of these terms). In practice, both of these effects are accounted for by performing a Doppler shift of the photon angle and energy between the different velocities at cell interfaces.

The fluid frame IMC radiation energy equation is operator split into two equations that describe the Lagrangian derivative of the photon intensity. The equations resulting from operator splitting are solved in the following order. The first describes the advection of the MC photons with the fluid:

$$
\frac{1}{c} \frac{\partial I}{\partial t}+\frac{1}{c} \bar{u} \cdot \bar{\nabla} I=0
$$

The second is a radiation energy equation without the Lagrangian time derivative:

$$
\begin{aligned}
\frac{1}{c} \frac{\partial I}{\partial t} & +\bar{\Omega} \cdot \bar{\nabla} I-\frac{1}{c} \bar{\Omega} \cdot \bar{\nabla} \bar{u} \cdot(\overline{\bar{l}}-\bar{\Omega} \bar{\Omega}) \cdot \bar{\nabla}_{\bar{\Omega}} I+\frac{3}{c} \bar{\Omega} \cdot \bar{\nabla} \bar{u} \cdot \bar{\Omega} I \\
& =\frac{1}{4 \pi} \kappa f a c T^{4}-\kappa I+\frac{1}{4 \pi} \int_{0}^{4 \pi} \kappa(1-f) I d \bar{\Omega}^{\prime} .
\end{aligned}
$$




\subsubsection{Implicit radiation diffusion}

It is convenient to define the zeroth angular moment of the radiative transfer equation [14]:

$$
\frac{D E}{D t}+\bar{\nabla} \cdot \bar{F}+\overline{\bar{p}}: \bar{\nabla} \bar{u}=\kappa f a c T^{4}-c \kappa f E
$$

where $\overline{\bar{p}_{r}}(\bar{r}, t)=\int_{0}^{4 \pi} \bar{\Omega} \bar{\Omega} I(\bar{r}, t, \bar{\Omega}) d \bar{\Omega}\left[\mathrm{N} / \mathrm{cm}^{2}\right]$ is the radiation pressure tensor. To this point we have only made a few assumptions: Eq. 8 is only accurate to $O\left(\frac{u}{c}\right)$ and the Fleck and Cummings approximation for the source emission term is $O(\Delta t)$. These assumptions are minor and are equally applicable to both transparent and opaque media. The next assumptions are more restrictive in terms of their accuracy in transparent media.

The diffusion approximation is commonly used to evaluate the radiation energy in optically thick diffusive material [11, 14]:

$$
\frac{D E}{D t}+c \bar{\nabla} \cdot \frac{1}{3 \kappa} \bar{\nabla} E+\frac{4}{3} E \bar{\nabla} \cdot \bar{u}=\kappa f a c T^{4}-c \kappa f E .
$$

The diffusion equation is developed applying some additional assumptions to the zeroth moment of the radiative transfer equation (Eq. 11). The first of these restrictive assumptions is that the radiation pressure tensor can be defined as a function of the energy density. This is know as the Eddington approximation [11]. The diffusion assumptions additionally require the flux to have negligible time dependence and the flux dotted into the gradient of the velocity to be negligible. These assumptions allow us to represent the flux using Ficks Law.

\subsubsection{Operator splitting radiation diffusion}

In diffusion the velocity terms in Eq. 12 are evaluated using operator splitting rather than Doppler shifting or an aberration correction as is done for IMC. Similar to IMC the material energy equation is operator split into the hydrodynamic terms (Eq. 6) and radiation terms (Eq. 7).

The remaining term in Eq. 12 that is difficult to evaluate is the velocity gradient. This term describes the work done to the radiation field by the movement of the fluid. The radiation hydrodynamic diffusion equation (Eq. 12) is operator split such that we are solving the stationary diffusion equation:

$$
\frac{\partial E}{\partial t}+c \bar{\nabla} \cdot \frac{1}{3 \kappa} \bar{\nabla} E=\kappa f a c T^{4}-c \kappa f E,
$$

and the hydrodynamic motion is evaluated separately with two different equations. We do this by expanding the Lagrangian derivative of the energy density, similar to what is done for IMC to form Eq. 9, resulting in the advection of the energy density:

$$
\frac{\partial E}{\partial t}+\bar{u} \cdot \bar{\nabla} E=0 .
$$

The remaining velocity terms are collected into the final equation that evaluate the work performed on the radiation field by the material motion:

$$
\frac{\partial E}{\partial t}+\frac{4}{3} E \bar{\nabla} \cdot \bar{u}=0
$$


It is possible to transform Eq. 15 by changing the spatial derivative of the velocity into a Lagrangian derivative of the volume $V$ :

$$
\frac{\partial E}{\partial t}+\frac{4}{3} \frac{E}{V} \frac{D V}{D t}=0 .
$$

This amounts to changing the photon energy density according to the change in the material volume.

The radiation diffusion and material energy equations (Eqs. 7 and 13) are now in their familiar stationary form with the addition of two extra equations: Eq. 14 evaluates the radiation advection with material and Eq. 16 evaluates the work done to the radiation by the material motion.

We will now describe how these radiation hydrodynamic equations, with their applicable assumptions, are evaluated using Lagrangian hydrodynamics and our HIMCD radiative transfer method.

\section{HIMCD and The Radiation Hydrodynamics Equations}

In this work we evaluate the radiation hydrodynamics equations, with their associated approximations developed in Section 1, in the following order. First the Lagrangian hydrodynamics physics package evaluates the conservation of mass, momentum, and material energy equations (Eqs. 1,3, and 6).

$$
\begin{gathered}
\frac{D \rho}{D t}+\rho \bar{\nabla} \cdot \bar{u}=0 \\
\rho \frac{D \bar{u}}{D t}+\bar{\nabla} p-\kappa \bar{F}=0 \\
\rho \frac{D \epsilon}{D t}+p \bar{\nabla} \cdot \bar{u}=0
\end{gathered}
$$

The total radiation momentum deposition $(\kappa \bar{F})$ is provided from the previous time step of the radiation physics package. After the Lagrangian hydrodynamics equations are solved the radiation field is evaluated using Monte Carlo.

HIMCD defines the radiation field over two discrete domains: a diffusion domain and a transport domain. IMD is used to evaluate the operator split diffusion equations (Eqs. 14, 16, and 13) in the cells that make up the diffusion domain.

$$
\begin{gathered}
\frac{\partial E}{\partial t}+\bar{u} \cdot \bar{\nabla} E=0 \\
\frac{\partial E}{\partial t}+\frac{4}{3} \frac{E}{V} \frac{D V}{D t}=0 \\
\frac{\partial E}{\partial t}+c \bar{\nabla} \cdot \frac{1}{3 \kappa} \bar{\nabla} E=\kappa f a c T^{4}-c \kappa f E
\end{gathered}
$$

IMC is used to evaluate the transport equations (Eqs. 9 and 10) in the remaining transport cells.

$$
\frac{1}{c} \frac{\partial I}{\partial t}+\bar{u} \cdot \bar{\nabla} I=0
$$




$$
\begin{aligned}
\frac{1}{c} \frac{\partial I}{\partial t} & +\bar{\Omega} \cdot \bar{\nabla} I-\frac{1}{c} \bar{\Omega} \cdot \bar{\nabla} \bar{u} \cdot(\overline{\bar{l}}-\bar{\Omega} \bar{\Omega}) \cdot \bar{\nabla}_{\bar{\Omega}} I+\frac{3}{c} \bar{\Omega} \cdot \bar{\nabla} \bar{u} \cdot \bar{\Omega} I \\
& =\frac{1}{4 \pi} \kappa f a c T^{4}-\kappa I+\frac{1}{4 \pi} \int_{0}^{4 \pi} \kappa(1-f) I \partial \bar{\Omega}^{\prime}
\end{aligned}
$$

These domains are evaluated simultaneously by allowing Monte Carlo particles to transition between the domains according to predefined interface conditions that are defined in the next section. After the HIMCD package evaluates the radiation fields the material energy is updated, to evaluate the radiation exchange with the material, using Eq. 7:

$$
\rho \frac{\partial \epsilon}{\partial t}=c \kappa f E-\kappa f a c T^{4}
$$

This ends the current time step, and all remaining time steps are evaluated in this same fashion.

Now we will discuss the temporal and spatial discretization used in both methods. The discretization choices directly affect the interface conditions that need to be defined between the diffusion and transport domain.

\subsection{Temporal discretization}

IMC and IMD treat time dependence slightly differently. IMC tracks Monte Carlo particles continuously in time while IMD tracks particles discretely in time using the backward Euler temporal discretization. The differences are highlighted by looking at the time integrated IMC equation:

$$
\begin{aligned}
\frac{1}{c} I^{n+1} & +\int_{t^{n}}^{t^{n+1}} \bar{\Omega} \cdot \bar{\nabla} I d t-\int_{t^{n}}^{t^{n+1}} \frac{1}{c} \bar{\Omega} \cdot \bar{\nabla} \bar{u} \cdot(\overline{\bar{l}} d t-\bar{\Omega} \bar{\Omega}) \cdot \bar{\nabla}_{\bar{\Omega}} I d t \\
& +\int_{t^{n}}^{t^{n+1}} \frac{3}{c} \bar{\Omega} \cdot \bar{\nabla} \bar{u} \cdot \bar{\Omega} I d t=\frac{1}{4 \pi} \kappa f a c T^{n 4} \Delta t-\int_{t^{n}}^{t^{n+1}} \kappa I d t \\
& +\int_{t^{n}}^{t^{n+1}} \frac{1}{4 \pi} \int_{0}^{4 \pi} \kappa(1-f) I \partial \bar{\Omega}^{\prime} d t+\frac{1}{c} I^{n}
\end{aligned}
$$

and the time integrated diffusion equation:

$$
E^{n+1}+c \bar{\nabla} \cdot \frac{1}{3 \kappa} \bar{\nabla} E^{n+1} \Delta t=\kappa f a c T^{n 4} \Delta t-c \kappa f E^{n+1} \Delta t+E^{n}
$$

where the superscripts $n$ and $n+1$ denote the initial and final simulation time of the time step $\Delta t=t^{n+1}-t^{n}$. We see that the time integrated IMC equation tracks photons continuously in time $\left(\int_{t^{n}}^{t^{n+1}} I(t) d t\right)$ and IMD uses a first order backward Euler discretization for time averaged values $\left(\int_{t^{n}}^{n^{n+1}} E(t) d t \approx E\left(t^{n+1}\right) \Delta t\right)$. The superscript $n$ denotes different time point locations. When an IMD particle transitions from the diffusion domain to the transport domain we must resample the particle time. 


\subsection{Spatial discretization}

IMC particles are tracked continuously in space as opposed to IMD particles that are tracked discretely in space. The discrete tracking of the IMD particles is completely dependent upon the diffusion discretization scheme used to develop the tracking probabilities [7].

Consider a single cell $i$ of a Cartesian mesh with a diffusion criterion $\gamma_{i}^{n}$ that has been pre-computed based on the cell's properties at the beginning of a time step (see Figure 1). This diffusion criterion will be used to determine if the radiation field will be evaluated with diffusion (IMD) $\left(\gamma_{i}^{n}=\right.$ true) or transport (IMC) $\left(\gamma_{i}^{n}=\right.$ false). The cell under consideration has $J_{i}+M_{i}$ faces, where there are $J_{i}$ internal faces (denoted individually by the variable $j$ ) and $M_{i}$ transport-diffusion faces (denoted individually by the variable $m$ ). An internal face in the transport domain is a face for which the diffusion criterion is false for both cells on either side of the face. A transport-diffusion face is a face for which the diffusion criterion is true for the cell on one side of the face but false for the cell on the other.

Applying a second order cell-centered finite volume discretization to the radiation diffusion equation (Eq. 13) for cell $i$ and integrating over a single time step yields the discretized form of the diffusion equation [15]:

$$
\begin{aligned}
E_{i}^{n+1} \Delta V_{i} & -c \sum_{j}^{J_{i}} \bar{n}_{j} \cdot D_{j} \nabla E_{j}^{n+1} \Delta t A_{j}-c \sum_{m}^{M_{i}} \int_{A} \int_{t^{n}}^{t^{n+1}} \bar{n}_{m} \cdot D_{m} \nabla E_{m} d t d A_{m} \\
& +c \kappa f E_{i}^{n+1}=\kappa f a c T^{n 4} \Delta t \Delta V+E_{i}^{n} \Delta V_{i}
\end{aligned}
$$

where $\bar{n}$ is the unit normal, $D$ is the diffusion coefficient, and $A$ is the area for the internal and diffusion face (subscripts $j$ and $m$ respectively). Coefficients associated with the transport-diffusion faces (denoted by $m$ subscript) are developed later in the diffusion-transport spatial interface condition and are therefore left in the integral form. See Appendix A for details on the development of face averaged diffusion coefficients and momentum deposition.

\subsection{Diffusion-transport interface conditions}

Monte Carlo particles can easily be tracked by either the IMC or IMD tracking procedures and only require additional information sampling (location, direction, and time) when they transition from IMD to IMC. The IMD algorithm simply disregards the extra information when MC particles are transitioned from IMC to IMD.

The transitions between IMC and IMD particles are based on the temporal and spatial interface conditions that couple the diffusion and transport domains. The diffusion and transport domains are defined using the spatial and time dependent diffusion criterion $\gamma(\bar{r}, t)$.

The diffusion criterion $\left(\gamma\left(\bar{r}_{i}, t^{n}\right)=\gamma_{i}^{n}\right)$ is pre-computed for every cell $(i)$ at the beginning of every time step $(n)$. Remember that we are employing frequency independent transport, and if frequency dependent transport were considered, the diffusion criterion would be dependent on frequency as well as position. 


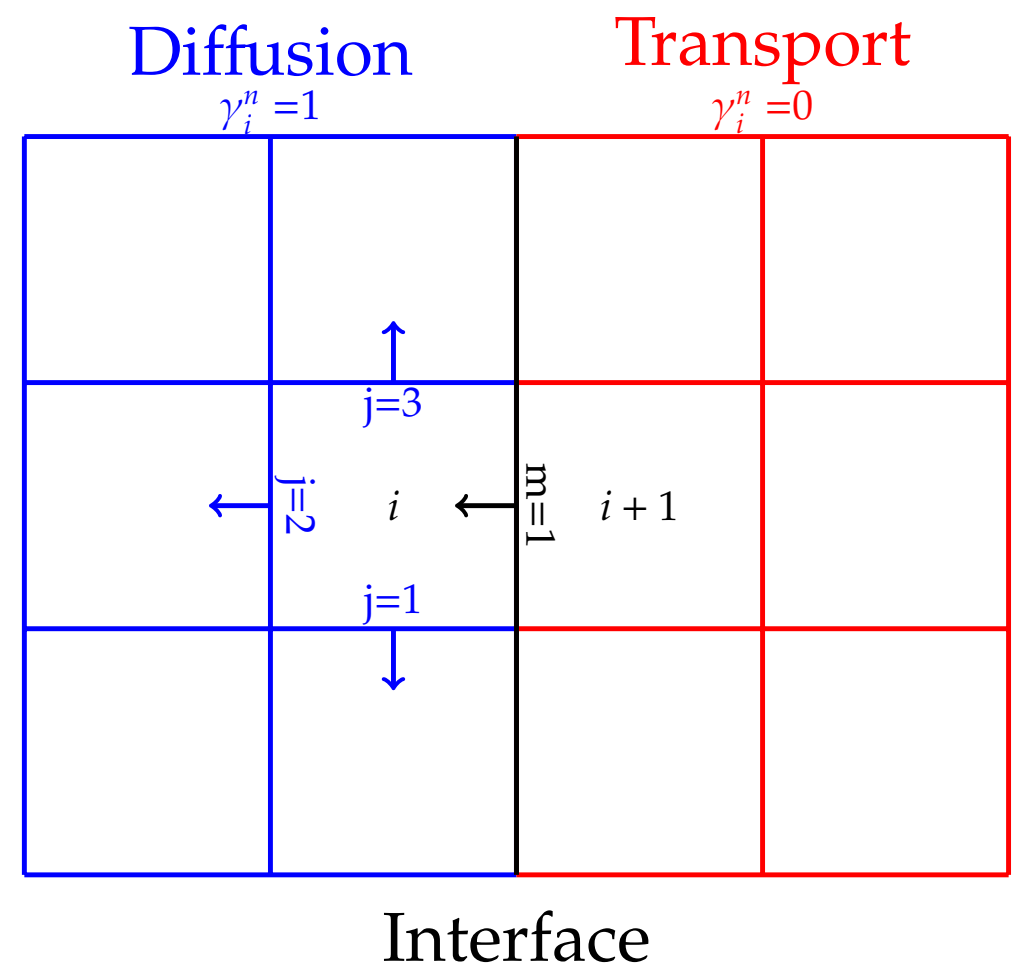

Figure 1: This figure shows a diffusion-transport interface on a simple Cartesian mesh. The diffusion zones are blue, the transport zones are red, and the diffusion-transport interface is black. The interior transport, or diffusion, cell faces are denoted by the variable $j$ and the face defining the diffusion transport interface is labeled as $m$. The $j$ and $m$ numbers are relative to each cell in this figure we show the local face numbering for both a diffusion cell and transport cell. These interface cells both have $J_{i}=3$ interior faces and $M_{i}=1$ diffusiontransport faces. The arrows in this figure show the normal direction of each face relative to a diffusion, or transport, cell. The interior face are all defined with an outward normal from the current cell center. The interface normal (shown by the black arrow) is fixed such that it always points from the transport zone to the diffusion zone. This means the interface has an inward normal from the perspective of a diffusion cell and an outward normal from the perspective of a transport cell. The cell center can be referenced by the global cell ID $i$.

\subsubsection{Diffusion-transport temporal interface condition}

We previously defined the diffusion criterion $\gamma_{i}^{n}$ as a function of both space and time to allow the diffusion, and consequently the transport, domain to adapt during the time dependent simulation. This means that any given cell $i$ can change between the diffusion and transport domain. To evaluate these transitions between the domains we will define a temporal interface condition to account for a transport cell becoming a diffusion cell:

$$
\left.\begin{array}{ll}
\text { if } & \gamma_{i}^{n-1}=0 \\
\text { and } & \gamma_{i}^{n}=1
\end{array}\right\} \quad E^{n}=\frac{1}{c} \int_{0}^{4 \pi} I^{n} d \bar{\Omega}
$$


or if a diffusion cell becomes a transport cell:

$$
\left.\begin{array}{ll}
\text { if } & \gamma_{i}^{n-1}=1 \\
\text { and } & \gamma_{i}^{n}=0
\end{array}\right\} I^{n}=\frac{1}{\Delta V} \frac{1}{4 \pi} c E^{n} \Delta V .
$$

Here we intentionally kept fractions that could be canceled out to show that, when a diffusion cell becomes a transport cell in a subsequent time step $\left(\gamma_{i}^{n-1}=1\right.$ and $\left.\gamma_{i}^{n}=0\right)$, we are required to redistribute the energy density in angle $\left((4 \pi)^{-1}\right)$ and space $\left((\Delta V)^{-1}\right)$. The assumption that the previous energy density $E^{n}$ is equally distributed in space and angle may incur a small amount of error because it neglects the spatial distribution of the energy density and the linear isotropic assumption made by the diffusion approximation.

\subsubsection{The diffusion-transport spatial interface condition}

We employ the diffusion transport interface condition originally developed by Densmore et al. [16] for an interface between IMC and DDMC. This interface interface condition is defined such that it will preserve an accurate emissivity at the interface regardless of cell size [16]. The emissivity is defined as the fraction of incident radiation that is not reflected back through the incident surface [17]. Densmore's boundary is derived in the fluid frame, and must be evaluated there.

The flux at the interface $F_{m}$ is used to define the diffusion-interface-cell matrix coefficients in Eq. 17:

$$
\left.\begin{array}{ll}
\text { if } & \gamma_{i}^{n}=1 \\
\text { and } & \gamma_{i+1}^{n}=0
\end{array}\right\} \int_{A} \int_{t^{n}}^{t^{n+1}} \bar{n}_{m} \cdot D_{m} \bar{\nabla} E_{m} d t d A_{m}=\int_{A} \int_{t^{n}}^{t^{n+1}} \bar{n}_{m} \cdot \bar{F}_{m} d t d A_{m} .
$$

The three terms that arise in the IMD coefficient matrix are treated as three separate physical process that occur at interface face $m$ : the IMC energy that enters the diffusion domain:

$$
\int_{A} \int_{t^{n}}^{t^{n+1}} \int_{0}^{2 \pi} \int_{0}^{1}\left(1-P_{\text {ref, }, \mathrm{m}}\left[\mu_{m}\right]\right) \mu_{m} I_{m} d \mu d \theta d t d A_{m}
$$

the IMD energy that diffuses out into the transport domain:

$$
c E_{i}^{n+1} C_{m} \Delta t A_{m},
$$

and the IMC energy that is reflected back into the transport domain:

$$
\int_{A} \int_{t^{n}}^{t^{n+1}} \int_{0}^{2 \pi} \int_{0}^{1} P_{\text {ref,m }}\left[\mu_{m}\right] \mu_{m} I_{m} d \mu d \theta d t d A_{m}
$$

The new terms, $P_{\text {ref,m }}\left[\mu_{m}\right]$ and $C_{m}$, result from applying Densmore's interface condition to the IMD equations. (See Appendix B for details). The matrix coefficient $C_{m}$ arises from applying the interface condition to the diffusion equations. $P_{\text {ref,m }}\left[\mu_{m}\right]$ is the probability that an IMC particle will reflect off of a diffusion-transport interface back into the transport cell.

The interface condition for a transport cell that adjoins the face $m$ can be broken down into two parts: one evaluating the photon intensity that is entering the cell $\left(\mu_{m}<0\right)$ and the other evaluating the photon intensity that leaves the cell $\left(\mu_{m}>0\right)$. The angular flux 
$\left(\left.\mu I\right|_{m}\right)$ entering the transport cell through the diffusion-transport face $(m)$ is defined such that (if $\mu_{m}<0$ ):

$$
\begin{aligned}
\left.\mu I\right|_{\mu_{m}<0} & =\frac{1}{A_{m}} \frac{1}{\Delta t} \frac{1}{2 \pi} \frac{\mu}{\int_{-1}^{0} \mu_{m} d \mu} c E_{i}^{n+1} C_{m} \Delta t A_{m} \\
& +\frac{1}{2 \pi} \frac{\mu}{\int_{-1}^{0} \mu_{m} d \mu} \int_{0}^{2 \pi} \int_{0}^{1} P_{\text {ref }, \mathrm{m}}\left[\mu_{m}\right] \mu_{m} I_{m} d \mu d \theta .
\end{aligned}
$$

We have intentionally left fractions that can be canceled in these equations to show that it is necessary to sample the particle direction $\left((2 \pi)^{-1}\right.$ and $\left.\mu\left(\int_{-1}^{0} \mu_{m} d \mu\right)^{-1}\right)$, location $\left(\left(A_{m}\right)^{-1}\right)$, and time $\left((\Delta t)^{-1}\right)$ when an IMC particle is transitioned to an IMD particle. Notice that it is not necessary to re-sample the particle's location or time when they are reflected back into the transport cell. These reflections are assumed to be instantaneous and occur where and when the IMC particle originally crossed the face $m$. The angular flux that leaves the transport cell through the face $m$ is defined as (if $\mu_{m}>0$ ):

$$
\left.\mu I\right|_{\mu_{m}>0}=\left(1-P_{\text {ref,m }}\left[\mu_{m}\right]\right) \mu_{m} I_{m}
$$

The overall interface condition can be written as a composition of the intensity leaving and entering the transport cell though the face $m$ :

$$
\left.\begin{array}{ll}
\text { if } & \gamma_{i}^{n}=0 \\
\text { and } & \gamma_{i+1}^{n}=1
\end{array}\right\}\left.\quad \mu I\right|_{m}=\left\{\begin{array}{lll}
\left.\mu I\right|_{\mu_{m}<0} & \text { if } & \mu_{m}<0 \\
\left.\mu I\right|_{\mu_{m}>0} & \text { if } & \mu_{m}>0
\end{array}\right.
$$

It is easy to show the this transport interface condition is consistent with the diffusion interface condition using the previously defined interface flux $F_{m}$ :

$$
\begin{aligned}
& \left.\int_{A} \int_{t^{n}}^{t^{n+1}} \int_{0}^{2 \pi} \int_{-1}^{1} \mu I\right|_{m} d \mu d \theta d t d A_{m} \\
= & \int_{A} \int_{t^{n}}^{t^{n+1}} \int_{0}^{2 \pi}\left(\left.\int_{-1}^{0} \mu I\right|_{\mu_{m}<0}+\left.\int_{0}^{1} \mu I\right|_{\mu_{m}>0}\right) d \mu d \theta d t d A_{m} \\
= & \int_{A} \int_{t^{n}}^{t^{n+1}} \bar{n}_{m} \cdot \bar{F}_{m} d t d A_{m}
\end{aligned}
$$

The reflection probability has a constraint on both the scattering ratio $(\omega)$ and cell optical thickness $(\tau)$ to insure $1 \leq P_{\text {ref }, m}[\mu] \leq 0$. These constraints are wrapped into the definition of the diffusion criterion.

\subsection{The diffusion criterion}

The diffusion criterion developed in this work adheres to the minimum constraints required to produce reflection probabilities that are bound between 1 and 0 for a diffusion transport interface [16]. The diffusion criterion is defined using each cell's minimum optical depth $\left(\tau_{\min }\right)$ and scattering ratio $(\omega)$. Though a lower scattering ratio can be used 
to generate valid interface probability ranges, a $90 \%$ scattering ratio was chosen in this work so that transitions to diffusion occur only when diffusion is valid.

$$
\gamma_{i}= \begin{cases}1 & \text { if } \omega \geq 0.9 \text { and } \tau_{\min } \geq 4 \\ 0 & \text { else }\end{cases}
$$

We evaluate this diffusion criterion for every cell at the beginning of every time step given the current mesh and material properties. The scattering ratio:

$$
\omega=\frac{\kappa_{s}+\kappa(1-f)}{\kappa+\kappa_{s}}
$$

is quickly and easily evaluated using the ratio of the scattering and total opacity ( $\kappa_{S}$ and $\left.\left(\kappa+\kappa_{s}\right)\right)$. In the absance of physical scattering $\kappa_{s}=0$. The optical depth is defined as:

$$
\tau_{\min }=\left(\kappa+\kappa_{s}\right) \bar{n} \cdot \overline{\Delta X}_{\min }
$$

where the vector $\overline{\Delta X}_{\min }=2\left(\bar{X}_{f}-\bar{X}_{i}\right)$ is two times the vector from the cell center $\bar{X}_{i}$ to the closest cell face $\bar{X}_{f}$. This is an approximation of the minimum cell width.

\subsection{Applying HIMCD to evaluate the radiation field}

At the beginning of the time step the diffusion criterion $\gamma_{i}^{n}$ is evaluated to define the diffusion domain $\left(\gamma_{i}^{n}=1\right)$. The IMD probabilities are generated using the matrix that forms the left hand side of the discretized diffusion equation (Eq. 17). Greater detail on how these probabilities are developed can be found in reference [7]. IMC and IMD particles are exchanged during Monte Carlo particle tracking according to the previously defined interface conditions.

The transition from an IMC particle to an IMD particle during the Monte Carlo particle tracking requires no additional information. This is because IMD particles only carry energy and an identifier that associates it with a diffusion cell. Therefore, upon the determination of a transport-diffusion transition Monte Carlo particles only need to be tracked using the IMD probabilities rather than the IMC tracking procedure.

It is more complicated for an IMD particle to be transitioned into an IMC particle. This is because the IMD particles carry no information with regard to their exact position, direction, and time. All of this information is needed in the IMC tracking procedure. There are two different diffusion-transport transitions that are evaluated using previously defined interface conditions: zone transitions and face transitions.

Zone diffusion-transport transitions only occur at the beginning of a time step, in the absence of frequency dependence, when a zone that was previously determined to be a diffusion zone is re-designated as a transport zone for the next time step (as defined by the temporal interface conditions defined in section 2.3.1). This means that the census information $E^{n}$ was originated by IMD particles and contains no information with respect to their exact position, direction, or time. We assume that energy density in the previous time step is equally distributed in angle and location. These new IMC particles are given the current time step's initial simulation time just as they would be in the case for an IMC only simulation.

Face diffusion-transport transitions occur during the Monte Carlo tracking procedure (as defined by the spatial interface conditions defined in section 2.3.2). These transitions 
require the selection of a new IMC particle direction, location, and simulation time. We choose to use a simple face source distribution to represent these transitions. Hence, transition photons are equally distributed over the area of the transition face. The transition photons are given a cosine angular distribution with respect to the face normal. These two assumptions should have little effect on the overall accuracy of the simulation. The final assumption is that these photons are distributed equally in time over the time step. This sampling would not be required in DDMC because of the explicit tracking of time. We argue that though this is an additional approximation it will have little effect on the overall accuracy of the HIMCD solution, as compared to the angular and spatial assumptions already made for the diffusion zones.

This work was implemented in the Kull [18] multiphysics software package that already contains previously documented IMC [18] and Lagrangian hydrodynamics [19] libraries to evaluate the radiation hydrodynamics equations. The IMD $[1,7]$ solver described in this work can be used as a stand alone radiation package in Kull. The HIMCD solver described in this work couples this new IMD solver to the IMC solver already present in Kull.

\section{Results}

This work investigates two different test cases: a semi-analytic radiating shock and a simple ablation test problem. Each test case is evaluated using the same Lagrangian hydrodynamics package coupled to one of 5 possible radiation packages: IMC, IMD, HIMCD, discrete ordinates $\left(S_{N}\right)$ [20], or flux limited diffusion (FLD) [21].

The radiating shock problem demonstrates that the fluid frame IMC-hydrodynamics and IMD-hydrodynamics methods can accurately produce the solution to coupled radiation hydrodynamics problems. This radiating shock problem can be accurately evaluated using diffusion throughout the spatial domain and therefore our HIMCD method shows very little improvement over using IMD. This problem does not test the HIMCD diffusion/interface because the problem specification prevents it from being implemented. That is, there is no cell face that will satisfy the diffusion-transport interface conditions. Thus we included a second ablation test case which demonstrates a problem where the diffusion/transport interface dynamically moves during the simulation.

The ablation test case shows the performance advantages of the HIMCD method as compared to IMC for a heterogeneous problem. This problem tests the ability of the diffusion criteria to dynamically isolate diffusive regions of the problem. This problem also tests the accuracy of the diffusion/transport interface condition, where particles transition from IMC to IMD or vise versa. The performance improvements come with little cost to accuracy because diffusion is only used in regions where its assumptions are valid. The $S_{N}$ solution provides a base numerical solution to compare the other radiation solvers. The FLD solution was generated to highlight some of the known deficiencies of evaluating the radiation field in optically thin materials using a diffusion method (IMD-only results are not presented because the simulation times were prohibitively long).

All simulations where performed on $2.8 \mathrm{GHz}$ Intel Xeon processors with $2 \mathrm{~GB}$ of memory per processor. 


\subsection{A semi-analytic radiation diffusion shock}

The semi-analytic radiation diffusion shock benchmark developed by Lowrie and Edwards [3] is used to verify the coupled IMD-hydrodynamics presented in this work. The radiating shock investigated in this work has a Mach number of 45 . The pre-shock and post-shock conditions are listed in Table 1 . The material is assumed to be a gamma

Table 1: Pre-shock and post-shock conditions.

\begin{tabular}{|l|l|l|l|}
\hline Properties & Pre-Shock & Post-Shock & Units \\
\hline$\rho$ & 1 & 6.4261 & {$[\mathrm{~g} / \mathrm{cc}]$} \\
$u$ & 0 & $-4.8175 \times 10^{8}$ & {$[\mathrm{~cm} / \mathrm{s}]$} \\
$T$ & 0.1 & 8.3578 & {$[\mathrm{keV}]$} \\
\hline
\end{tabular}

law gas. The gamma law gas assumption provides a closure for Eq. 7. The problem has homogeneous materials whose properties are listed in Table 2.

Table 2: Simulation material properties.

\begin{tabular}{|c|c|c|}
\hline Properties & Values & Units \\
\hline$\kappa$ & $5.0940 \times 10^{8} \rho T^{-3.5}$ & {$\left[\mathrm{~cm}^{2} / \mathrm{g}\right]$} \\
$\kappa_{s}$ & 0.4006 & {$\left[\mathrm{~cm}^{2} / \mathrm{g}\right]$} \\
$\gamma$ & $5.0 / 3.0$ & Unitless \\
$C_{v}$ & $0.1447 \times 10^{15}$ & {$[\mathrm{erg} /(\mathrm{g} \mathrm{keV})]$} \\
\hline
\end{tabular}

The shock front is driven by a black body emission source with the post-shock material temperature on the right most boundary face. The initial sonic point, where the material Mach number is equal to 1 , is set at $x=2300[\mathrm{~cm}]$. The initial conditions for the simulations are defined using Lowrie's analytic result. This initial analytic profile is fully converged and should not change as the wave propagates through the material. The numerical shock profile should be directly comparable to the analytic profile translated by the known shock velocity.

The shock simulation was performed on an initially equally spaced 1D mesh consisting of 5000 zones spanning the domain $0 \leq x \leq 2500[\mathrm{~cm}]$ using $1 \times 10^{7}$ Monte Carlo particles per time step. The spatial zoning used makes most cells optically thin particularly in the hot regions of the problem. The simulation was evaluated from the initial time $t=0$ to a final time $t=1.5 \times 10^{-7}[\mathrm{sec}]$ using a maximum time step size of $1 \times 10^{-10}$ [sec]. Each simulation was mesh replicated across 64 processors.

Figures 2, 3, and 4 show that IMC, IMD, and HIMCD all produce accurate solutions compared to the analytic answer. Of the three methods the IMD simulation was found to be the fastest. The total number of Monte Carlo paths, the simulation times, and their associated speed up, compared to IMC, are shown in Table 3. A Monte Carlo path consists of one movement of a Monte Carlo particle to either a material interaction position, a census position, or a cell face. IMD takes more paths than IMC because the heated cells are optically thin making the IMD leakage probability large. This in effect creates many scattering-like events (that is, particles moving back and forth between 


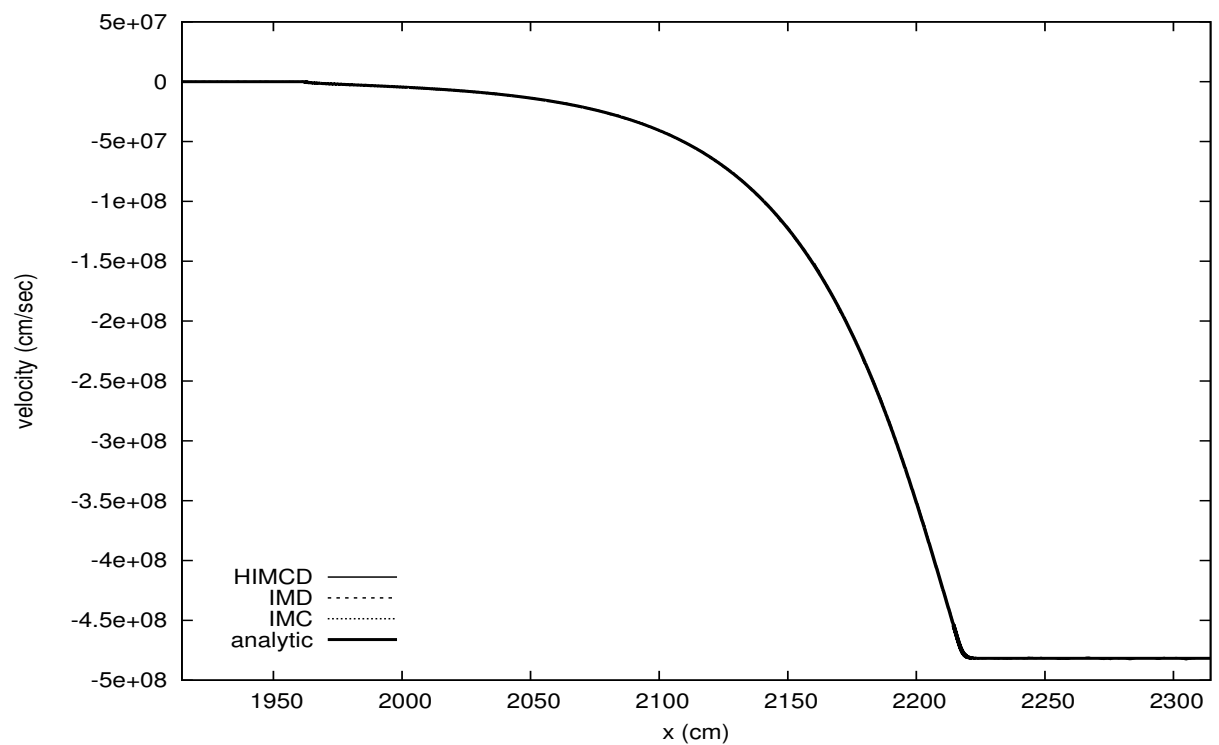

Figure 2: Velocity profiles for the Mach 45 shock simulation at $1.5 \times 10^{-7}$ [sec] as evaluated separately by the IMD, IMC, and HIMCD algorithms compared to the analytic result

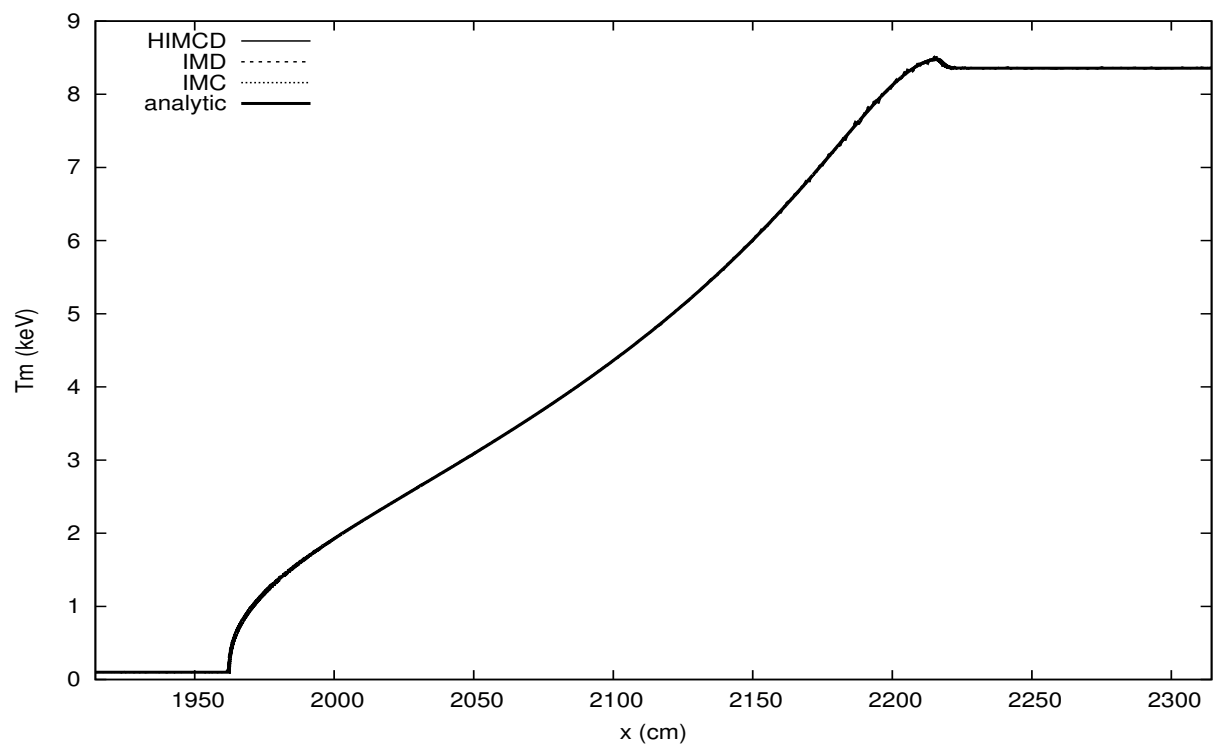

Figure 3: Material temperature ( $\mathrm{Tm})$ profiles for the Mach 45 shock simulation at $1.5 \times 10^{-7}$ [sec] as evaluated separately by the IMD, IMC, and HIMCD algorithms compared to the analytic result

cells while depositing very little energy). The number of IMC paths taken by the HIMCD match with the number taken by the pure IMC simulation. Zero IMD paths were taken by HIMCD because the diffusion criterion was never valid during the simulation. The 


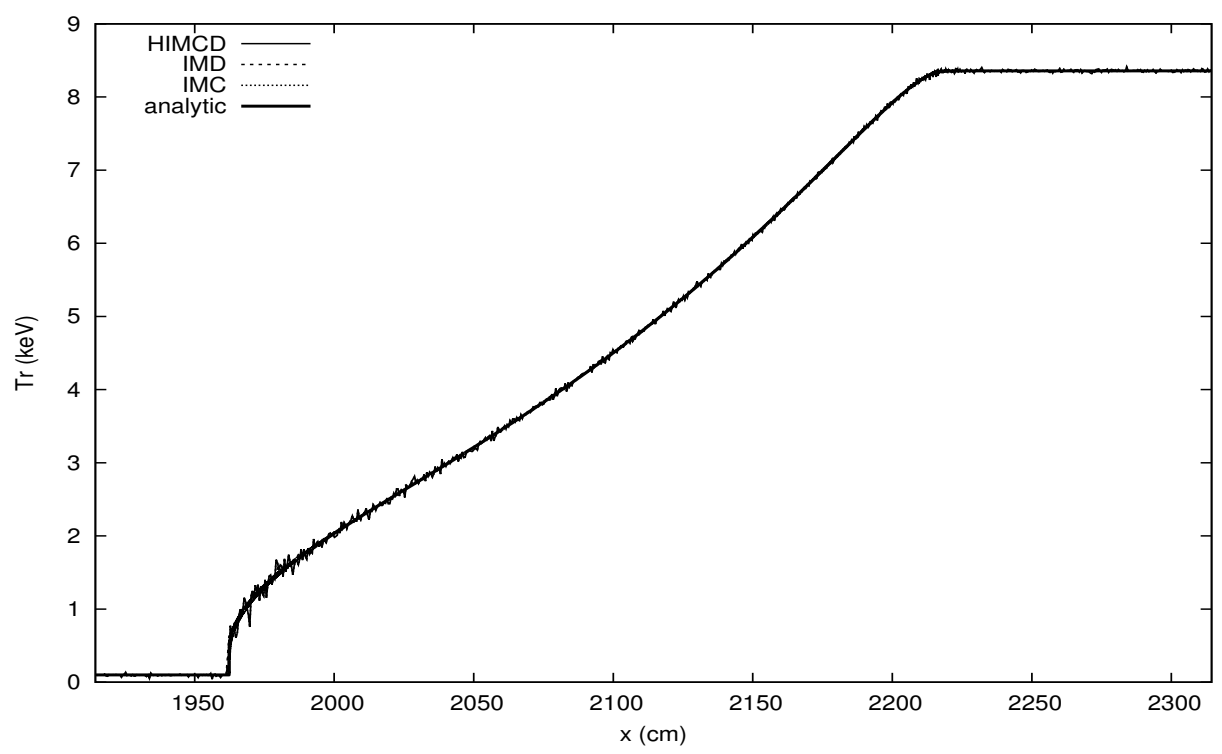

Figure 4: Radiation temperature (Tr) profiles for the Mach 45 simulation at $1.5 \times 10^{-7}$ [sec] as evaluated separately by the IMD, IMC, and HIMCD algorithms compared to the analytic result

Table 3: Radiating shock run times

\begin{tabular}{|c|c|c|c|c|}
\hline Method & time [min] & speed up & \multicolumn{2}{|c|}{ \# of paths } \\
& & & IMC & IMD \\
\hline IMC & 68 & 1 & $2 \times 10^{11}$ & N/A \\
IMD & 40 & 1.7 & N/A & $6 \times 10^{11}$ \\
HIMCD & 75 & 0.9 & $2 \times 10^{11}$ & 0.0 \\
\hline
\end{tabular}

fine spatial zoning prevented the HIMCD method from using IMD for any zones in the problem. This is because the cold region has an insufficient scattering ratio for the diffusion criterion to be true, and the hot zones, where the scattering ratio is very high, are not optically thick enough for the diffusion criterion to be true. This is an artifact of the spatial zoning used for this problem. Larger zones could be used for the post shock region of the problem, which would increase the optical thickness of the zones there.

The HIMCD simulation time was slightly longer than the IMC simulation time even though they took the same number of paths. This is because the HIMCD simulation still evaluated the IMD interaction probabilities at the beginning of every time step. The HIMCD method dynamically picked the least number of paths taken by each method. This turns out to be not ideal for this problem because the cost of an IMD path is much less than an IMC path. This is highlighted by the fact that even though the IMC simulation took significantly less paths it still had a slightly greater overall run time than the IMD simulation. The IMD simulation also produced less statistical noise than the IMC method, which would improve its figure of merit over the IMC simulation. 


\subsection{A simple ablation test problem}

The second test case is a simple ablation problem that consists of optically thick and optically thin material. The optically thick material, iron, spans the domain $0 \leq x \leq 5$ $[\mathrm{cm}]$ and the optically thin material, silicon dioxide, spans the remainder of the spatial domain $5 \leq x \leq 50[\mathrm{~cm}]$. The iron is separated into 150 mesh zones that geometrically increase in size from the material interface at $x=5[\mathrm{~cm}]$ to $x=0[\mathrm{~cm}]$. The silicon dioxide is composed of 50 spatial zones that geometrically increase in size from the material interface $x=5[\mathrm{~cm}]$ to $x=50[\mathrm{~cm}]$. The material starts at an initial temperature of $T=0.1[\mathrm{keV}]$ and is heated during the simulation by a $T_{s}=1.0[\mathrm{keV}]$ isotropic radiation source incident on the right side of the thin material at $x=50[\mathrm{~cm}]$. The relationship between the change in material energy to the change in material temperature (Eq. 7) is evaluated using the Livermore equation of state database for iron and silicon dioxide. The iron is given an initial density of $\rho_{\mathrm{Fe}}=7.86[\mathrm{~g} / \mathrm{cc}]$ and the silicon dioxide is given an initial density of $\rho_{\mathrm{SiO}_{2}}=2.65 \times 10^{-4}[\mathrm{~g} / \mathrm{cc}]$. The simulation was evaluated from the initial time $t=0$ to a final time $t=1 \times 10^{-7}$ [sec] using a maximum time step size of $1 \times 10^{-10}$ [sec]. Each method used $1 \times 10^{7}$ Monte Carlo particles per time step

During this simulation the radiation streams through the optically thin silicon dioxide and is absorbed by the optically thick iron. This causes the heated iron to rapidly expand, creating a shock front in both the iron and the silicon dioxide. There is no analytic solution to this problem and so we performed a direct comparison to three well documented solvers available in the Kull [18] multiphysics software package: discrete ordinates deterministic solver $\left(\mathrm{S}_{N}\right)$ [20], IMC [1], and flux limited diffusion (FLD) [21]. A FLD solution was generated in lieu of IMD for this test case because IMD is prohibitively expensive to evaluate in the hot thin materials where leakage probabilities are extremely high. We expect FLD to suffer from similar accuracy issues that IMD would in the optically thin regions of the problem. In fact FLD is slightly more accurate than IMD in these regions because the flux limiter prevents unphysically fast radiation propagation effects [22].

The IMC and HIMCD simulations were replicated over 128 processors. The FLD and $S_{N}$ simulations were only run on a single processor. The $S_{N}$ solvers used a $S_{6}$ level symmetric quadrature set. The FLD method used the Palmer nodal spatial discretization [21] with the Larsen flux limiter [23]. The $S_{N}$ solution was taken as the reference solution for this problem, because the problem does not have an analytic solution.

We found that the HIMCD, $S_{N}$, and IMC simulations match very well. Figures 5 and 6 show the radiation temperature at the end of the simulation and its corresponding relative error as compared to the $S_{N}$ reference solution. The FLD simulation produces an accurate radiation energy profile (see Figure 5). However the density, velocity, and material temperature profiles are not correct. Figures 7, 9, and 11 show the material density, velocity, and temperature profiles produced by each of the solution algorithms. The corresponding relative errors as compared to the $S_{N}$ solution can be seen in Figures 8 , 10 , and 12 . Looking at the material property profiles and their corresponding errors show that $S_{N}, I M C$, and HIMCD all agree to within at least $5 \%$ of each other, while the FLD solution deviates as much as $90 \%$.

FLD has significant relative errors, exceeding $50 \%$, for the material properties in the optically thin material. This is related to assumptions that are used in diffusion that are not applicable in thin material. Even though the energy density in the optically 


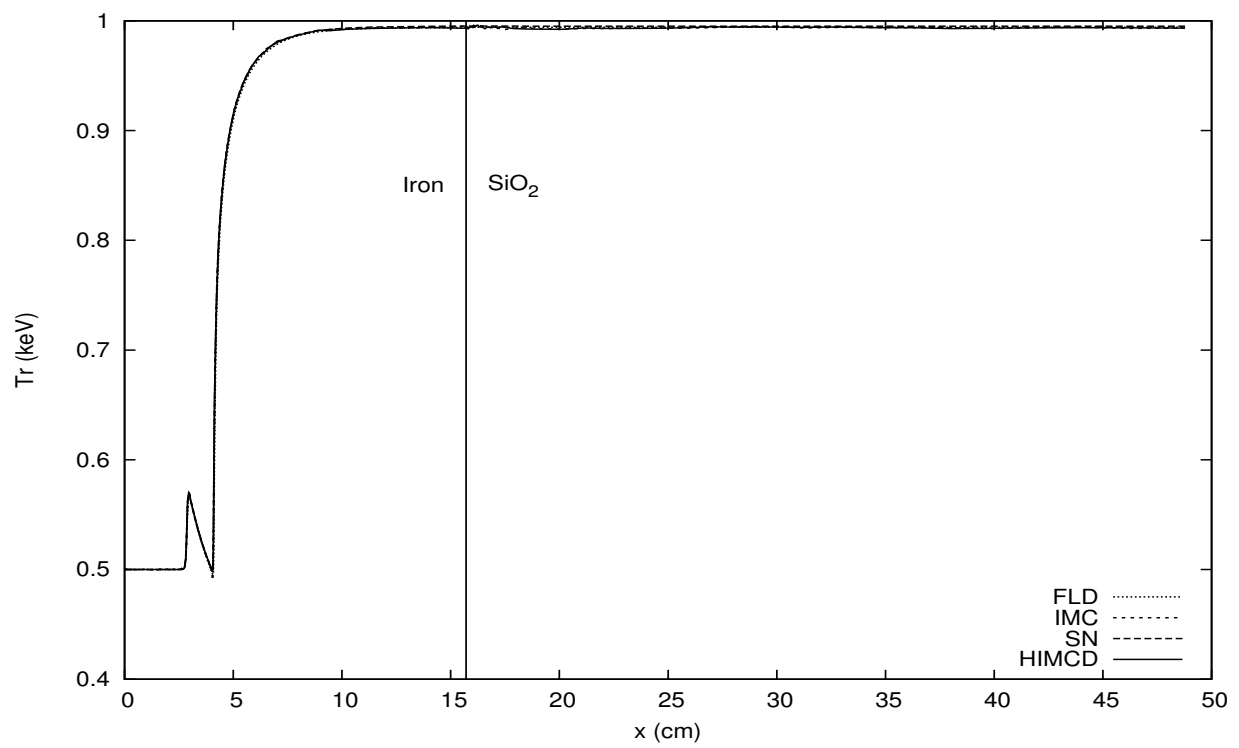

Figure 5: Radiation temperature ( $\mathrm{Tr}$ ) profiles for the ablation test case at $1 \times 10^{-7}$ [sec] as evaluated separately by the HIMCD, IMC, $S_{N}$, and FLD algorithms

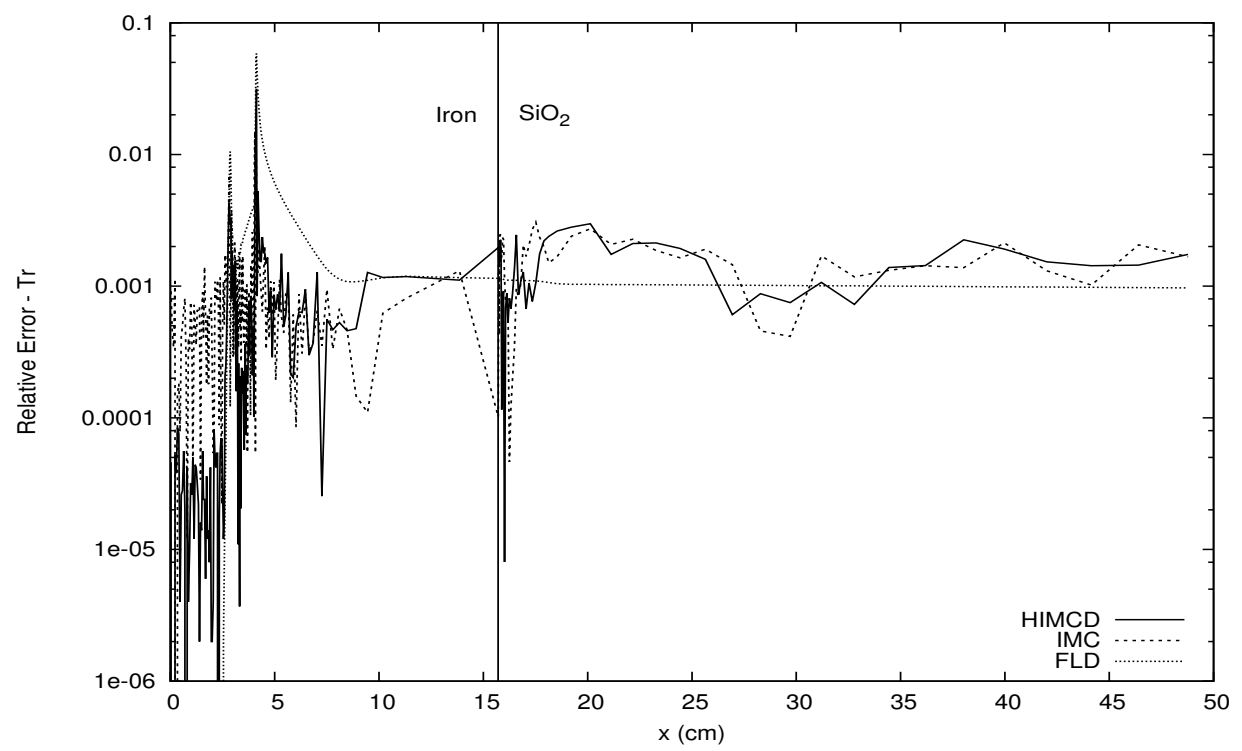

Figure 6: The relative error of the radiation temperature (Tr) for the ablation test case at $1 \times 10^{-7}$ [sec]. The $\mathrm{S}_{N}$ simulation was used as the reference solution to compare the accuracy of the HIMCD, IMC, and FLD methods.

thin material is flat, the photon intensity has a strong left peak from the heated right boundary condition. This flat energy density causes the diffusion streaming operator 
to push radiation equally in both directions artificially leaking excesses energy out the heated boundary. This reduction in radiation flow causes the shock timing to significant differ from the transport solution.

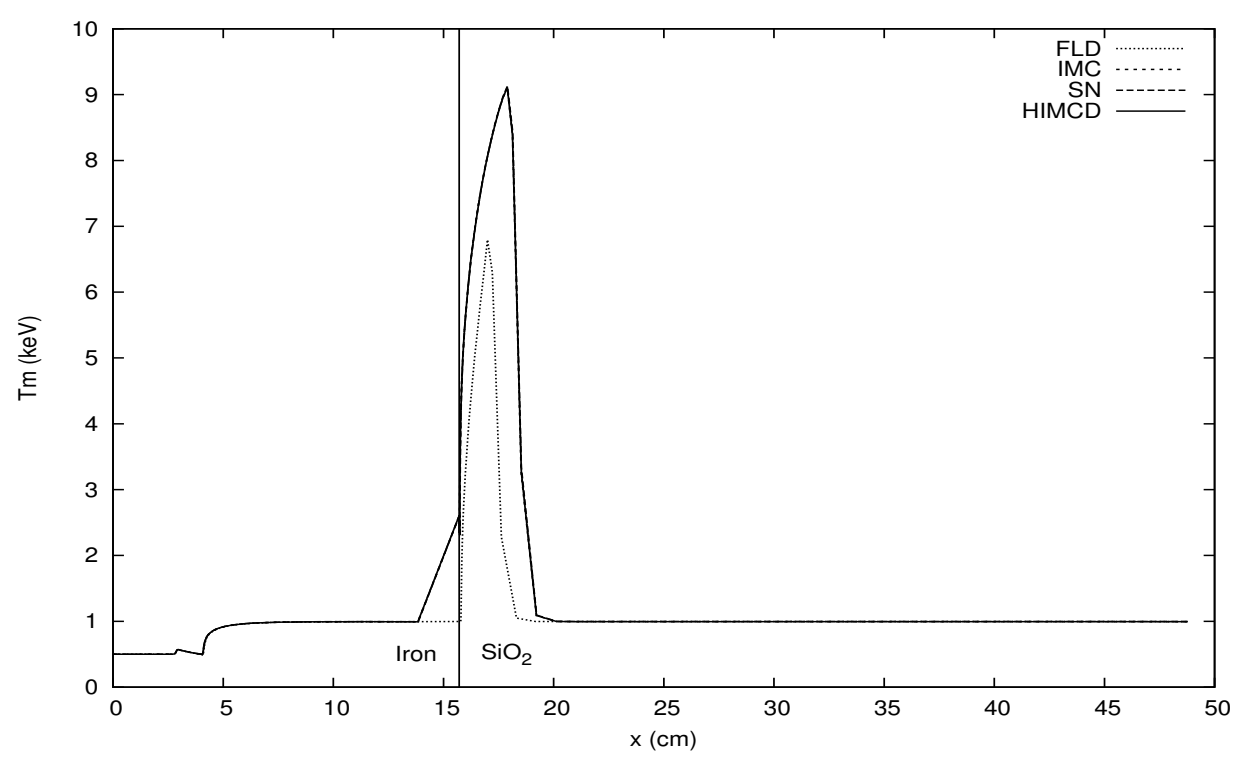

Figure 7: Material temperature (Tm) profiles for the ablation test case at $1 \times 10^{-7}$ [sec] as evaluated separately by the HIMCD, IMC, $S_{N}$, and FLD algorithms

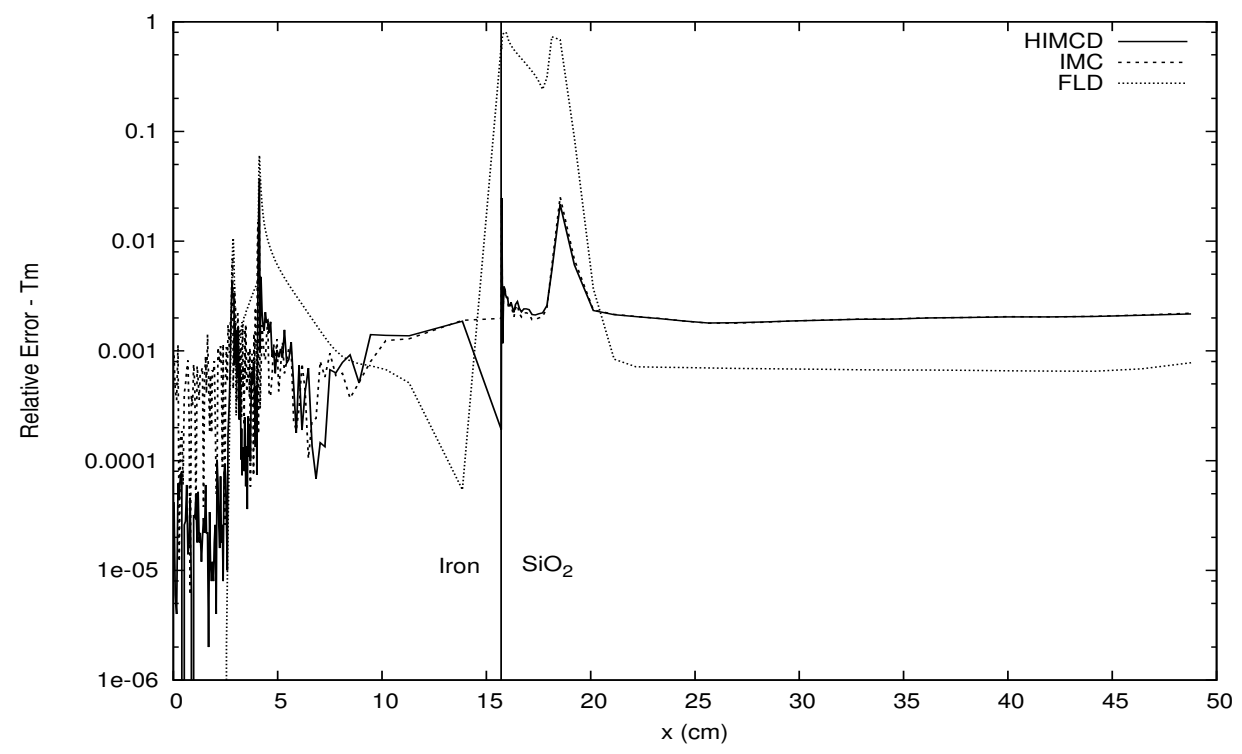

Figure 8: The relative error of the material temperature $(\mathrm{Tm})$ for the ablation test case at $1 \times 10^{-7}$ [sec]. The $\mathrm{S}_{N}$ simulation was used as the reference solution to compare the accuracy of the HIMCD, IMC, and FLD methods. 


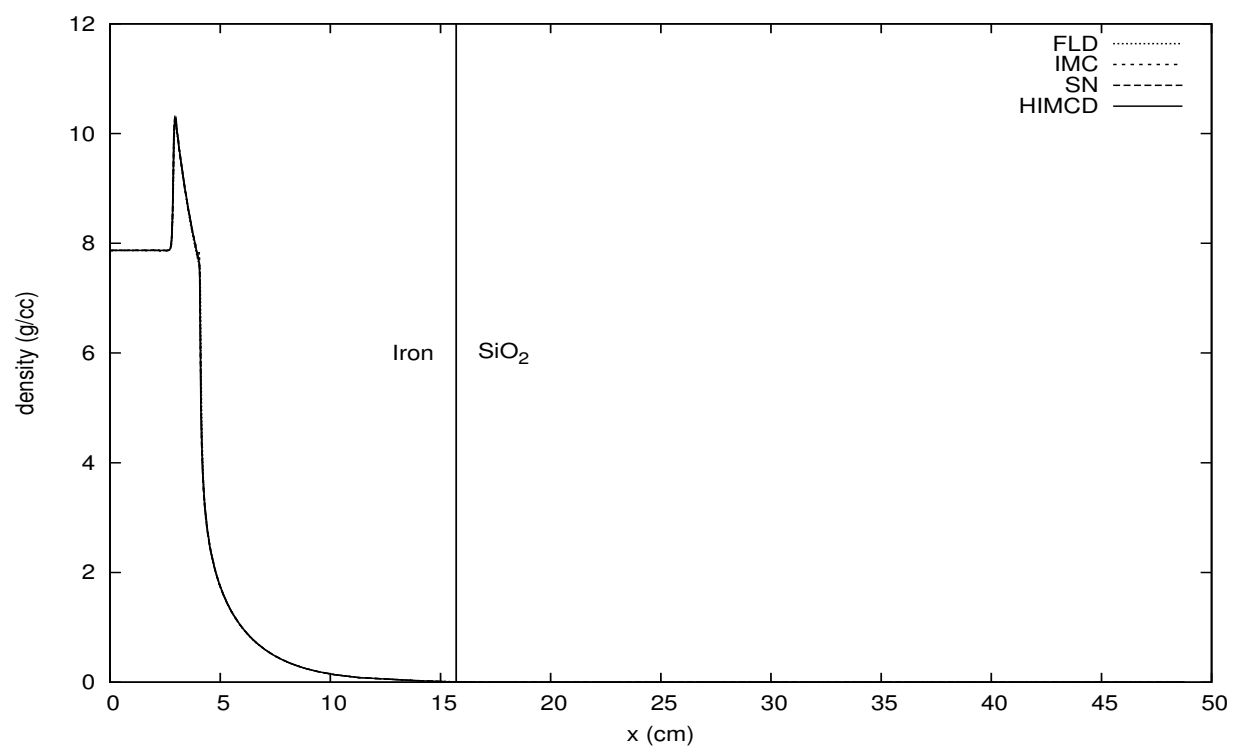

Figure 9: Material density profiles for the ablation test case at $1 \times 10^{-7}$ [sec] as evaluated separately by the HIMCD, IMC, $S_{N}$, and FLD algorithms

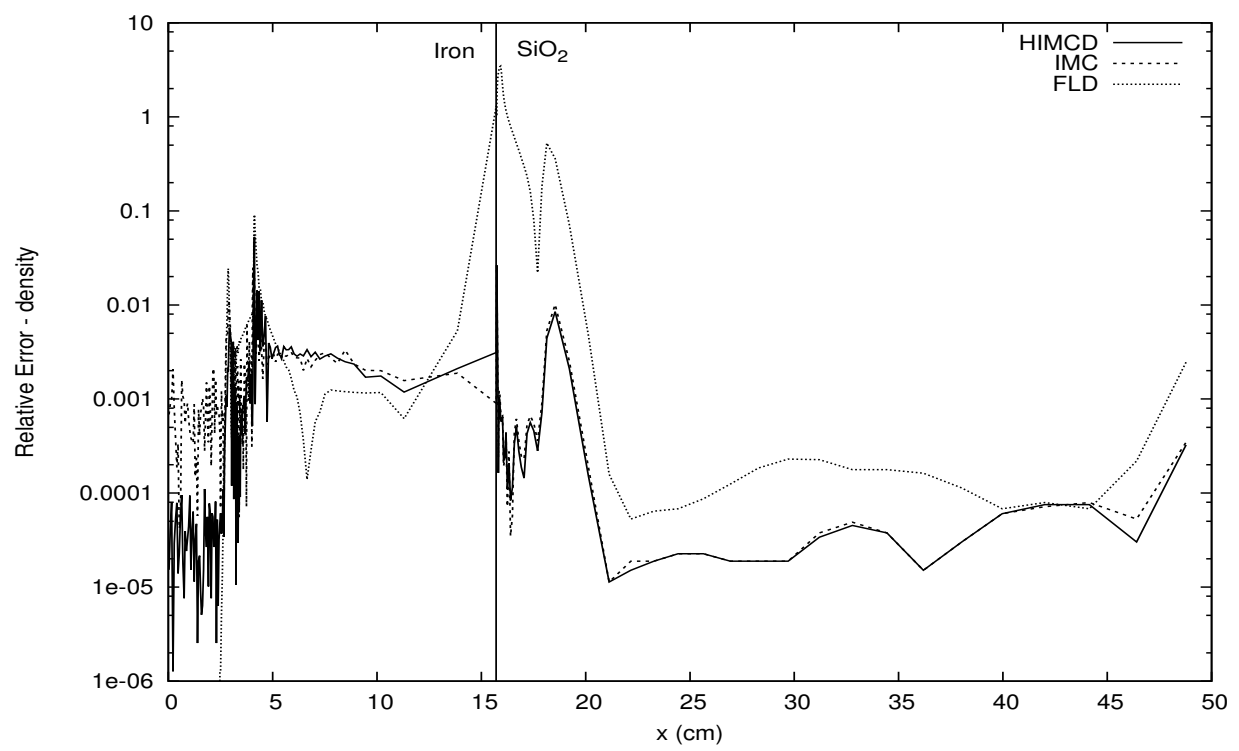

Figure 10: The relative error of the material density for the ablation test case at $1 \times 10^{-7}$ [sec]. The $\mathbf{S}_{N}$ simulation was used as the reference solution to compare the accuracy of the HIMCD, IMC, and FLD methods.

The simulation times shown in Table 4 indicate that the HIMCD method is 11 times faster than IMC. This speed-up can be directly related to the reduction of Monte Carlo paths taken in the opaque iron material. The total number of IMC and IMD paths are 


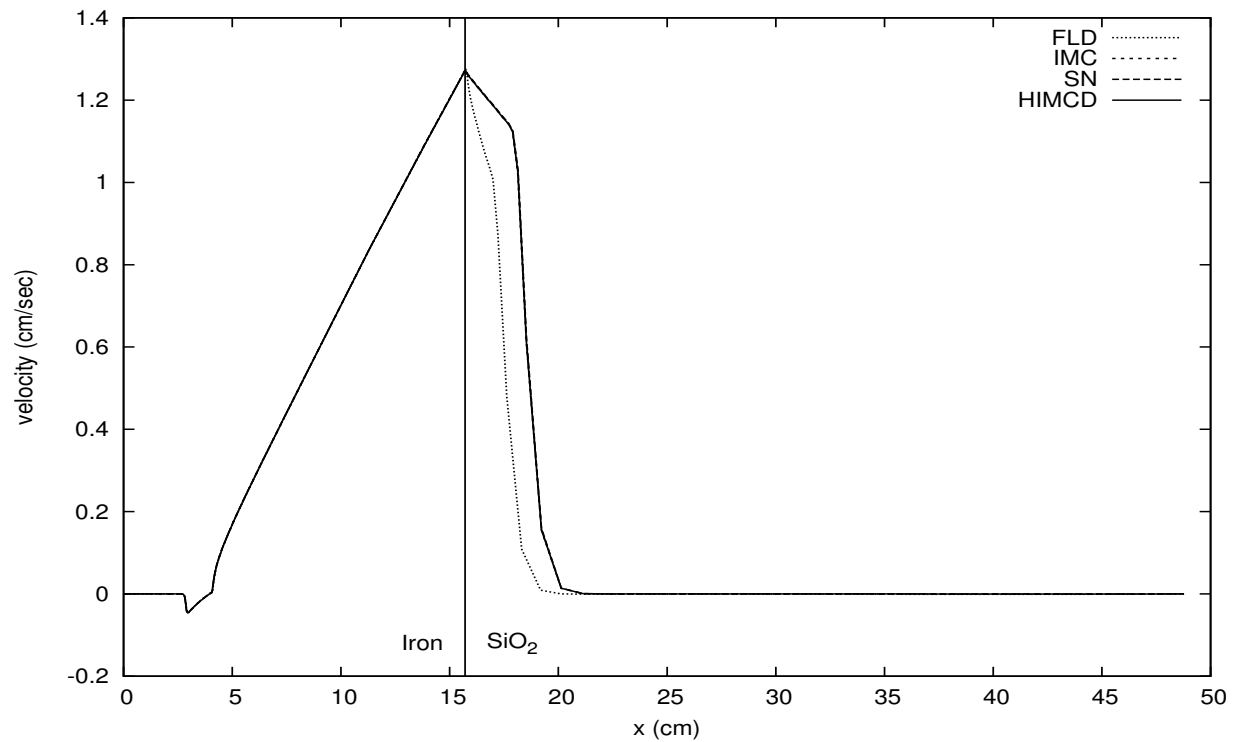

Figure 11: Material velocity profiles for the ablation test case at $1 \times 10^{-7}$ [sec] as evaluated separately by the HIMCD, IMC, $S_{N}$, and FLD algorithms

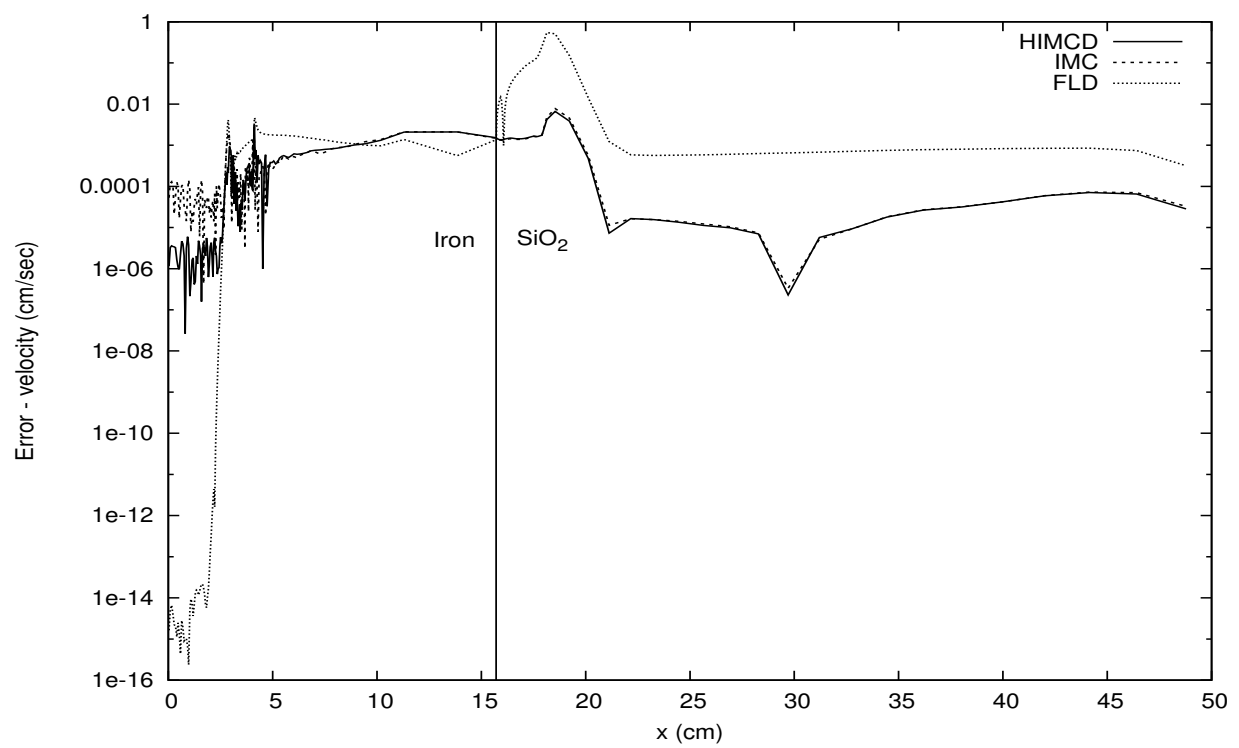

Figure 12: The absolute error of the material velocity for the ablation test case at $1 \times 10^{-7}$ [sec]. The $S_{N}$ simulation was used as the reference solution to compare the accuracy of the HIMCD, IMC, and FLD methods.

listed in Table 4, and a spatial distribution of the total paths is shown in Figure 13. This spatial distribution show that the HIMCD simulation replaces the large number of IMC 
paths in the optically thick diffusive material with approximately 100 times fewer IMD paths. This reduction in total Monte Carlo paths is the source of the HIMCD speed up.

Table 4: Ablation test case run times

\begin{tabular}{|c|c|c|c|c|}
\hline Method & time [min] & speed up & \multicolumn{2}{|c|}{ \# of paths } \\
& & & IMC & IMD \\
\hline IMC-128p & 232 & 1 & $2 \times 10^{12}$ & N/A \\
HIMCD-128p & 21 & 11 & $1 \times 10^{11}$ & $2 \times 10^{10}$ \\
\hline
\end{tabular}

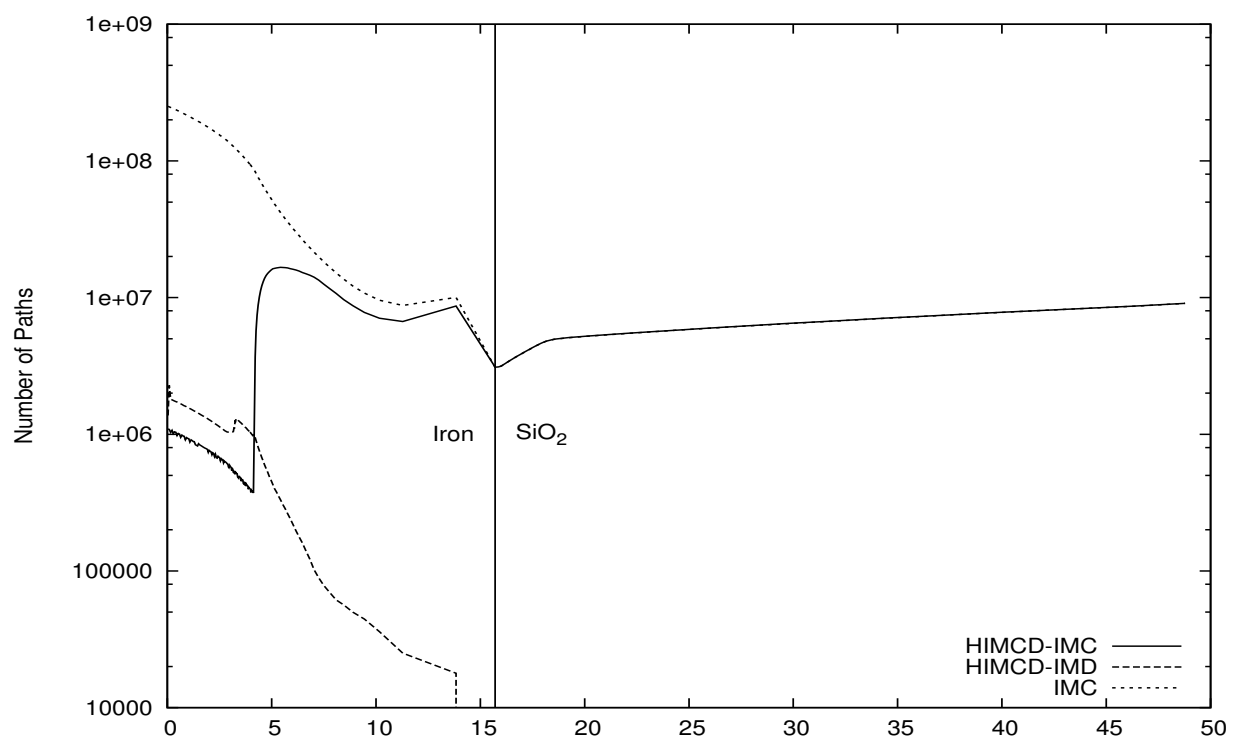

Figure 13: The total Monte Carlo path spatial distribution for the ablation test case at $1 \times 10^{-7}$ [sec]. The HIMCD simulation uses IMD paths (denoted as HIMCD-IMD) to evaluate the solution in the diffusive region of the problem and IMC paths (denoted by HIMCD-IMC) to evaluate the streaming region. The HIMCD simulation is compared against the pure IMC simulation.

\section{Conclusions}

In this work we outlined the implementation of a Hybrid Implicit Monte Carlo Diffusion algorithm that can be coupled to the Lagrangian hydrodynamics equations. This HIMCD algorithm applies the Implicit Monte Carlo Diffusion equations in regions of the problem that are dynamically determined to be optically thick and diffusive while applying Implicit Monte Carlo elsewhere in the problem. Monte Carlo particles are transitioned between the IMD and IMC radiation fields during the simulation given the transport-diffusion transition interface conditions defined in Section 2.3.

HIMCD, IMC, and IMD fluid frame radiation hydrodynamics algorithms accurately reproduced the semi-analytic diffusive shock problem (see Figures 2, 3, and 4). For 
this problem our diffusion criterion (defined in Section 2.4) for the HIMCD approach was never satisfied for any of the cells during the simulation. This may be surprising considering the semi-analytic solution is generated using diffusion theory. The hot (post shock) cells do indeed meet the scattering requirements defined by the HIMCD diffusion criterion, exceeding $C_{s}=0.9$, but they were only a fraction of a mean free path thick. Consequently the small optical depth in the hot cells, and small scattering ratio in the cold cells, prevented the diffusion criterion from being met for the HIMCD algorithm and created high leakage probabilities for the IMD algorithm. The high leakage probabilities resulted in many more paths for the IMD algorithm as compared to IMC. Even though the IMD algorithm was faster than the IMC solution (see Table 3), it took 6 times the number of paths to resolve the solution. Coarsening the spatial mesh could improve the efficiency of both the IMD and HIMCD method. However, it will come at the cost of decreased accuracy, because the discretization scheme is only second order in space. The cold regions (pre-shock) cells never approached a scattering ratio that would accommodate the diffusion criterion. The HIMCD solution took slightly longer to evaluate the same exact solution as the IMC algorithm. This extra time comes from the need to evaluate the IMD probabilities that are subsequently ignored because the diffusion criterion is not met.

The Lowrie shock problem demonstrates an underlining limitation to the current, and all previous attempts, at defining a dynamic diffusion-transport interface condition. The current implementation of the emissivity preserving boundary condition [16] limits the optical thickness of any diffusion cell to ensure that the interface transition probablities are positive. Perhaps future work should consider adapting the emissivity conserving interface condition to be positive regardless of cell optical thickness. Diffusion cells could be determined using local length scales rather than cell size. This would allow for a diffusion-transport interface to be defined in the middle of the Lowrie Shock front to more rigorously test the interface condition.

HIMCD showed significant improvements in run time over the pure IMC and IMD methods for the ablation test case. The $\mathrm{S}_{N}$ solution was used as the reference solution for the ablation test case because there is no analytic solution. A FLD solution was generated in lieu of IMD for this test case because IMD is prohibitively expensive to evaluate in the hot optically thin materials where leakage probabilities are extremely high. The $S_{N}, I M C$, and HIMCD solution all agree to within 5\%. The HIMCD algorithm proved to be 11 times faster than standard IMC for this problem. This speed up was obtained without the cost of lost accuracy in the thin region of the problem. The HIMCD algorithm improves the speed of the IMC simulation by replacing most of the IMC paths in the optically thick iron with IMD paths. The only reason that any IMC paths are taken in the optically thick region of the iron is because we used a variable time step size. The scattering ratios for the smallest time steps do not meet the requirement $\omega=0.9$ of our diffusion criterion.

The fluid frame HIMCD equations produced accurate solutions to coupled radiation hydrodynamics problems. The HIMCD method proved to be more robust than IMD and faster than IMC. This is best highlighted in problems that contain sufficiently optically diffusive cells. The drawbacks of the HIMCD method are primarily associated with limitations of discretization schemes on specific mesh types [7] and slightly increased simulation times on problems that do not contain a significant amount of hot optically diffusive cells. Future work will investigate frequency dependent problems and more 
sophisticated discretization schemes for unstructured meshes.

\section{Appendix A. The diffusion coefficient and momentum deposition}

The face averaged diffusion coefficient for a second order finite volume discretization applied to orthogonal meshes can be expressed as [15]:

$$
D_{j}=\frac{D_{j+\frac{1}{2}} D_{j-\frac{1}{2}}\left(X_{j+\frac{1}{2}}-X_{j-\frac{1}{2}}\right)}{D_{j+\frac{1}{2}}\left(X_{j}-X_{j-\frac{1}{2}}\right)+D_{j-\frac{1}{2}}\left(X_{j+\frac{1}{2}}-X_{j}\right)}
$$

where $X$ is a position in space. The subscripts $\left(j+\frac{1}{2}\right)$ and $\left(j-\frac{1}{2}\right)$ denote cell-centered values on either side of the face.

The selection of the face averaged diffusion coefficient for this discretization scheme is important, because very thick cells can nonphysically stop photon propagation when adjoined by relatively thin cells [15]. This can be prevented, when the opacities are strongly temperature dependent, by weighting the cell diffusion coefficients with the material temperature. This weighting is such that the diffusion coefficient always favors the hot (or thin) material opacity:

$$
D_{j+\frac{1}{2}}^{\prime}=D_{j+\frac{1}{2}} \frac{T_{j+\frac{1}{2}}^{4}+T_{j-\frac{1}{2}}^{4}}{2 T_{j+\frac{1}{2}}^{4}} .
$$

The new material temperature weighted face averaged diffusion coefficient can be constructed by replacing all diffusion coefficients $D$ in Eq. A.1 with the weighted diffusion coefficient $D^{\prime}$.

Given the discretized diffusion equation, we still require a way to evaluate the radiation momentum deposition into the material $(\kappa \bar{F})$ in Eq. 3 . The radiation momentum deposition can be directly evaluated in the IMC simulation during particle tracking. This is not as straightforward for a diffusion scheme that can only evaluate the radiation flux at the cell faces. In this work we approximated the momentum deposition at the cell centers by averaging the momentum deposition at the face where the radiation flux is known, identical to the approach of Densmore et al. [5]:

$$
\kappa \bar{F}_{D, i} \approx \frac{\sum_{j}^{J} \kappa_{j} \bar{F}_{j} A_{j}+\sum_{m}^{M} \kappa_{m} \bar{F}_{D, m} A_{m}}{\sum_{j}^{J} \bar{n}_{j} A_{j}+\sum_{m}^{M} \bar{n}_{m} A_{m}}
$$

where the face average opacity is defined as:

$$
\kappa_{j}=\frac{1}{3 D_{j}^{\prime}} .
$$

\section{Appendix B. The diffusion-transport interface probabilities}

The incident partial flux $f^{+}$can be defined as a function of photon energy density at a diffusion face by using an asymptotic diffusion-limit interface condition [24]:

$$
f^{+}=\int_{0}^{1} \mu I d \mu=2 \int_{0}^{1} \mu I\left(1+\frac{3}{2} \mu\right) d \mu=c E-c \frac{\lambda}{\kappa} \bar{n} \cdot \bar{\nabla} E
$$


where $\lambda=0.7104$ is the extrapolation distance and $\mu=\bar{\Omega} \cdot \bar{n}$ is the cosine of the angle between the particle direction and the face unit normal $\bar{n}$. By applying the asymptotic diffusion-limit interface condition, Densmore et al. [16] defined the analytic emissivity $\epsilon^{\prime}$ for a face between two distinct semi-infinite slabs of materials each at fixed material temperatures, one diffusive and one a black body [16]:

$$
\epsilon^{\prime}=\frac{4}{3} \frac{\sqrt{3(1-\omega)}}{1+\lambda \sqrt{3(1-\omega)}}
$$

where $\omega=\frac{(1-f) \kappa}{\kappa}$ is the scattering ratio of the diffusive material. Note that the scattering $\left(\kappa_{s}=(1-f) \kappa\right)$ arises from the effective scattering that is formed out of the Fleck and Cummings IMC discretization. This diffusion criterion is used to form an expression for the partial fluxes at the interface $m$ assuming an equally spaced simple Cartesian geometry where we have defined a consistent unit normal (pointing from the transport cell to the diffusion cell) for all $M$ faces (see Figure 1). We have applied a second order finite volume spatial discretization in the development of this interface condition. The partial flux $\left(f_{m}^{+}\right.$and $\left.f_{m}^{-}\right)$for face $m$ can be expressed as:

$$
f_{m}^{+}=P_{m}^{\prime} \int_{0}^{2 \pi} \int_{0}^{1} \mu_{m} I_{m} d \mu d \theta
$$

and

$$
f_{m}^{-}=c E_{m+\frac{1}{2}}^{n+1} \frac{P_{m}^{\prime}}{4}+\left(1-P_{m}^{\prime}\right) \int_{0}^{2 \pi} \int_{0}^{1} \mu_{m} I_{m} d \mu d \theta
$$

where $P_{m}^{\prime}$ is defined such that it preserves the analytic emissivity $\epsilon^{\prime}$ and is a function of the diffusion interface cell's material properties and discretized mesh [16]:

$$
P_{m}^{\prime}=\frac{\epsilon^{\prime} \beta_{m}}{\beta_{m}-\frac{4}{3} \epsilon^{\prime} \tau_{m}}
$$

where the remaining variables are defined in reference [16] as:

$$
\begin{aligned}
\beta_{m}=\frac{3}{2}\left(1-\omega_{m}\right) \tau_{m}^{2} & +\sqrt{3\left(1-\omega_{m}\right) \tau_{m}^{2}+\frac{9}{4}\left(1-\omega_{m}\right)^{2} \tau_{m}^{4}} \\
\tau_{m} & =\kappa_{m+\frac{1}{2}} \bar{n}_{m} \cdot \overline{\Delta X} \\
\omega_{m} & =\frac{\kappa_{m+\frac{1}{2}}\left(1-f_{m+\frac{1}{2}}\right)}{\kappa_{m+\frac{1}{2}}}
\end{aligned}
$$

and $\overline{\Delta X}=2\left(\bar{X}_{m}-\bar{X}_{m+\frac{1}{2}}\right)$ is two times the vector from the position of the cell center $\bar{X}_{m+\frac{1}{2}}$ to the face center $\bar{X}_{m}$. We will refer to $\omega_{m}$ as the scattering ratio.

Given the definitions of the partial fluxes (Eqs. B.3 and B.4), we can define the total flux at the face as:

$$
\begin{aligned}
F_{m} & =f_{m}^{+}-f_{m}^{-} \\
& =\left(1-P_{\text {ref, } \mathrm{m}}\right) \int_{0}^{2 \pi} \int_{0}^{1} \mu_{m} I_{m} d \mu d \theta-c E_{m+\frac{1}{2}}^{n+1} C_{m}-P_{\text {ref }, \mathrm{m}} \int_{0}^{2 \pi} \int_{0}^{1} \mu_{m} I_{m} d \mu d \theta
\end{aligned}
$$


where we have defined two new variables: the diffusion interface leakage coefficient $C_{m}$ :

$$
C_{m}=\frac{P_{m}^{\prime}}{4}
$$

and reflection probability $P_{\text {ref }, m}$ :

$$
P_{\mathrm{ref}, m}=\frac{\int_{0}^{1} \mu_{m} I_{m} P_{\mathrm{ref}, m}\left[\mu_{m}\right] d \mu}{\int_{0}^{1} \mu_{m} I_{m} d \mu}=\frac{\int_{0}^{1} \mu_{m} I_{m}\left(1-P_{m}^{\prime}\right) 2\left(1+\frac{3}{2} \mu_{m}\right) d \mu}{\int_{0}^{1} \mu_{m} I_{m} d \mu} .
$$

The incident cosine angle-dependent reflection probability:

$$
P_{\text {ref }, m}\left[\mu_{m}\right]=\left(1-P_{m}^{\prime}\right) 2\left(1+\frac{3}{2} \mu_{m}\right)
$$

and the associated multiplication factor $\left(2\left(1+\frac{3}{2} \mu\right)\right)$ arise from substituting the asymptotic diffusion-limit interface condition (Eq. B.1) in for the definition of the incident flux $f^{+}$. The total flux $F_{m}$ can now be used to calculate the interface conditions between the transport and diffusion domains.

\section{Acknowledgments}

This work performed under the auspices of the U.S. Department of Energy by Lawrence Livermore National Laboratory under Contract DE-AC52-07NA27344.

\section{References}

[1] N. Gentile, Implicit Monte Carlo Diffusion: An acceleration method for Monte Carlo time-dependent radiative transfer simulations, Journal of Computational Physics 172 (2001) 543-571.

[2] J. Fleck Jr., J. Cummings Jr., An implicit Monte Carlo scheme for calculating time and frequency dependent nonlinear radiation transport, Journal of Computational Physics 8 (1971) 313-342.

[3] R. Lowrie, J. Edwards, Radiative shock solutions with grey nonequilibrium diffusion, Shock Waves 18 (2008) 129-143.

[4] J. Fleck Jr., E. Canfield, A random walk procedure for improving the computational efficiency of the implicit Monte Carlo method for nonlinear radiation transport, Journal of Computational Physics 54 (1984) 508-523.

[5] J. D. Densmore, T. J. Urbatsch, T. M. Evans, M. W. Buksas, A hybrid transport-diffusion method for Monte Carlo radiative-transfer simulations, Journal of Computational Physics 222 (2007) 485-503.

[6] E. Larsen, G. Pomraning, V. Badham, Asymptotic analysis of radiative transfer problems, Journal of Quantitative Spectroscopy and Radiative Transfer 29 (1983) 285-310.

[7] M. A. Cleveland, N. A. Gentile, T. S. Palmer, An extension of implicit Monte Carlo diffusion: Multigroup and the difference formulation, Journal of Computational Physics 229 (2010) 5707-5723.

[8] J. D. Densmore, T. J. Urbatsch, T. M. Evans, M. W. Buksas, Discrete Diffusion Monte Carlo for grey Implicit Monte Carlo simulations., Technical Report LA-UR-05-3781, Los Alamos National Laboratory, 2005.

[9] J. D. Densmore, G. Davidson, D. B. Carrington, Emissivity of discretized diffusion problems, Annals of Nuclear Energy 33 (2006) 583-593.

[10] J. D. Densmore, K. G. Thompson, T. J. Urbatsch, A hybrid transport-diffusion Monte Carlo method for frequency-dependent radiative-transfer simulations, Journal of Computational Physics 231 (2012) 6924-6934.

[11] J. I. Castor, Radiation Hydrodynamics, Cambridge University Press, 2007.

[12] J. Morel, Comoving-frame and laboratory-frame nonequilibrium grey radiation diffusion approximations in the non-relativistic limit, Presentation at the Conference on Numerical Methods for Multimaterial Fluid Flows, Prague, CZ, 2007. 
[13] D. Mihalas, B. Weibel-Mihalas, Foundations of radiation hydrodynamics, Courier Dover Publications, 1999.

[14] R. Buchler, Radiation transfer in the fluid frame, Journal of Quantitative Spectroscopy and Radiative Transfer 30 (1983) 395-407.

[15] R. H. Szilard, G. C. Pomraning, Numerical transport and diffusion methods in radiative transfer, 1992.

[16] J. D. Densmore, Interface methods for hybrid Monte Carlo-diffusion radiation-transport simulations, Annals of Nuclear Energy 33 (2006) 343-353.

[17] M. F. Modest, Radiative heat transfer, Academic Press, 2003.

[18] N. Gentile, N. Keen, J. Rathkopf, The Kull IMC package, Technical Report UCRL-JC-132743, Lawrence Livermore National Laboratory (LLNL), Livermore, CA, 1998.

[19] D. Miller, G. Zimmerman, An algorithm for time evolving volume fractions in mixed zones in lagrangian hydrodynamics calculations, Russian Journal of Physical Chemistry B, Focus on Physics 3 (2009) 117-121.

[20] P. Nowak, M. K. Nemanic, Radiation transport calculations on unstructured grids using a spatially decomposed and threaded algorithm, in: Proc. ANS Conf. on Math. and Computation, Reactor Phys. and Environmental Analysis in Nuclear Apps (1999), pp. 379-390.

[21] T. S. Palmer, Discretizing the diffusion equation on unstructured polygonal meshes in two dimensions, Annals of Nuclear Energy 28 (2001) 1851-1880.

[22] G. Pomraning, Flux limiters and eddington factors, Journal of Quantitative Spectroscopy and Radiative Transfer 27 (1982) 517-530.

[23] J. Morel, Diffusion-limit asymptotics of the transport equation, the p1/3 equations, and two flux-limited diffusion theories, Journal of Quantitative Spectroscopy and Radiative Transfer 65 (2000) 769-778.

[24] G. J. Habetler, B. J. Matkowsky, Uniform asymptotic expansions in transport theory with small mean free paths, and the diffusion approximation, Journal of Mathematical Physics 16 (1975) 846-854. 